\title{
Selenium as a pleiotropic agent for medical discovery and drug delivery
}

This article was published in the following Dove Press journal:

International Journal of Nanomedicine

\author{
Baozhang Guan ${ }^{1, *}$ \\ Ruiling Yan ${ }^{2, *}$ \\ Ruiman $\mathrm{Li}^{3}$ \\ Xingwang Zhang ${ }^{4}$ \\ 'Department of Nephrology, \\ The First Affiliated Hospital of Jinan \\ University, Guangzhou, China; ${ }^{2}$ Fetal \\ Medicine Department, The First \\ Affiliated Hospital of Jinan University, \\ Guangzhou, China; ${ }^{3}$ Department of \\ Gynecology and Obstetrics, The First \\ Affiliated Hospital of Jinan University, \\ Guangzhou, China; ${ }^{4}$ Department of \\ Pharmaceutics, College of Pharmacy, \\ Jinan University, Guangzhou, China \\ *These authors contributed equally \\ to this work
}

\begin{abstract}
Selenium as a biologically active element lends much support to health maintenance and disease prevention. It is now presenting pleiotropic effects on therapy and drug delivery. In this study, a profiling on the physiological functions, therapeutic significances, clinical/preclinical performances, and biomedical and drug delivery applications of selenium in different modalities was carried out. Major interests focused on selenium-based nanomedicines in confronting various diseases pertaining to selenium or not, especially in antitumor and antidiabetes. Furthermore, the article exclusively discusses selenium nanoparticles featured by ameliorative functions with emphasis on their applications in medical practice and drug delivery. The state-of-the-art in medical discovery as well as research and development on selenium and nano-selenium is discussed in this review.
\end{abstract}

Keywords: selenium, selenium nanoparticles, nanomedicine, biomedical applications, drug delivery, oncotherapy, diabetes

\section{Introduction}

Selenium is a trace element that is essential for human health. In recent years, the health benefits of selenium have been gradually recognized. ${ }^{1}$ Deficiency of nutrient selenium frequently results in the incidence of various disorders, such as diabetes, Keshan disease, thyroid dysfunction, arthrophyma, and cognitive limitation. ${ }^{2}$ Natural selenium is present in the soil and assimilated into various plants, including seeds, nuts, vegetables, and mushrooms. The human body receives selenium through the dietary intake of selenium-rich foodstuffs. The selenium content in the soil is geographically distributed unevenly on the global horizon. Selenium deficiency approximately affects 500 million to 1 billion people worldwide.

Selenium exists in the human body in the form of selenoproteins, which can be derived from ingested organic or inorganic selenium. Organic selenium (mainly selenomethionine) and ionic selenium (eg, selenite and selenate) are highly bioavailable, but elemental selenium is difficult to absorb by the gastrointestinal tract unless nanosized. ${ }^{3}$ Organic selenium from foods is relatively safe for the human body, whereas the inorganic one supplemented with chemicals exhibits a narrow window from therapeutic effect to toxic effect. ${ }^{4}$ Once being absorbed into the circulatory system, selenium will bind with various proteins in vivo to form Se/protein complexes, ie, selenoproteins, to exert the physiological actions. However, residents in the area that lacks selenium have the difficulty to assimilate enough naturally occurring selenium from the daily diet. Hence, some diseases associated with selenium deficiency occur. ${ }^{5-9}$ For these people, selenium supplementation becomes a crucial measure, which needs to be performed. Currently, selenium is not only utilized as a nutritional supplement for 
disease prevention and treatment but also applied for drug delivery in the form of nanoparticles to potentiate the carried therapeuticals. It is often delighted to anticipate a synergistic effect occurring between selenium and the payload. ${ }^{10,11}$

Selenium as a pleiotropic agent has attracted considerable attention for biotherapy and drug delivery. In particular, significant progresses in nanotechnology greatly escalate the translation of Se-based nanomedicines. In this study, we carried out a systematic literature review concerning selenium and discussed its physiological functions, therapeutic potential against relevant diseases, Se-based nanomedicines for cancer, and diabetes mellitus (DM) as well as selenium nanoparticles (SeNPs) for various medical purposes. Special interests focused on the pharmaceutical applications of SeNPs as drug delivery carriers with the highlight of encouraging outcomes.

\section{Physiological functions of selenium}

Selenium is a trace element that is essential for the body to carry out functions such as growth, metabolism, and hormone balance (Figure 1). Selenium is mostly famous for its antioxidative effect; it can serve as an antioxidant to remedy diseases relevant to oxidative stress, ${ }^{12}$ such as DM, atherosclerosis, and fatty liver. The effect of selenium on oxidative stress is realized by selenoproteins. ${ }^{13}$ Selenoproteins are proteins that include a selenocysteine ( $\mathrm{Sec}$ ) residue. Approximately 25 kinds of different selenoproteins have been observed in human cells and tissues so far. Among functionally characterized selenoproteins, there are five glutathione peroxidases (GPx) and three thioredoxin reductases (TrxR) with one Sec residue in structure. Selenoprotein P (SEPP) that contains $10 \mathrm{Sec}$ residues is the most common selenoprotein found in the plasma. The lack of selenium deprives the ability of the cells to synthesize selenoproteins; therefore, many health effects of low selenium intake are believed to be related to the absence of one or more specific selenoproteins. ${ }^{14}$ Residents in seleniumrich areas tend to have healthy bodies. The reaction of selenium with reactive oxygen more readily takes place in vivo and is reversible even under mild oxidative conditions. ${ }^{15}$ Various reactions involving selenium are described as "easy in-easy out". This is attributed to the strong nucleophilicity of selenium that can attack electrophiles. Meanwhile, the reactions are reversible due to the strong electrophilicity of selenium and the weakness of the selenium-carbon bond.

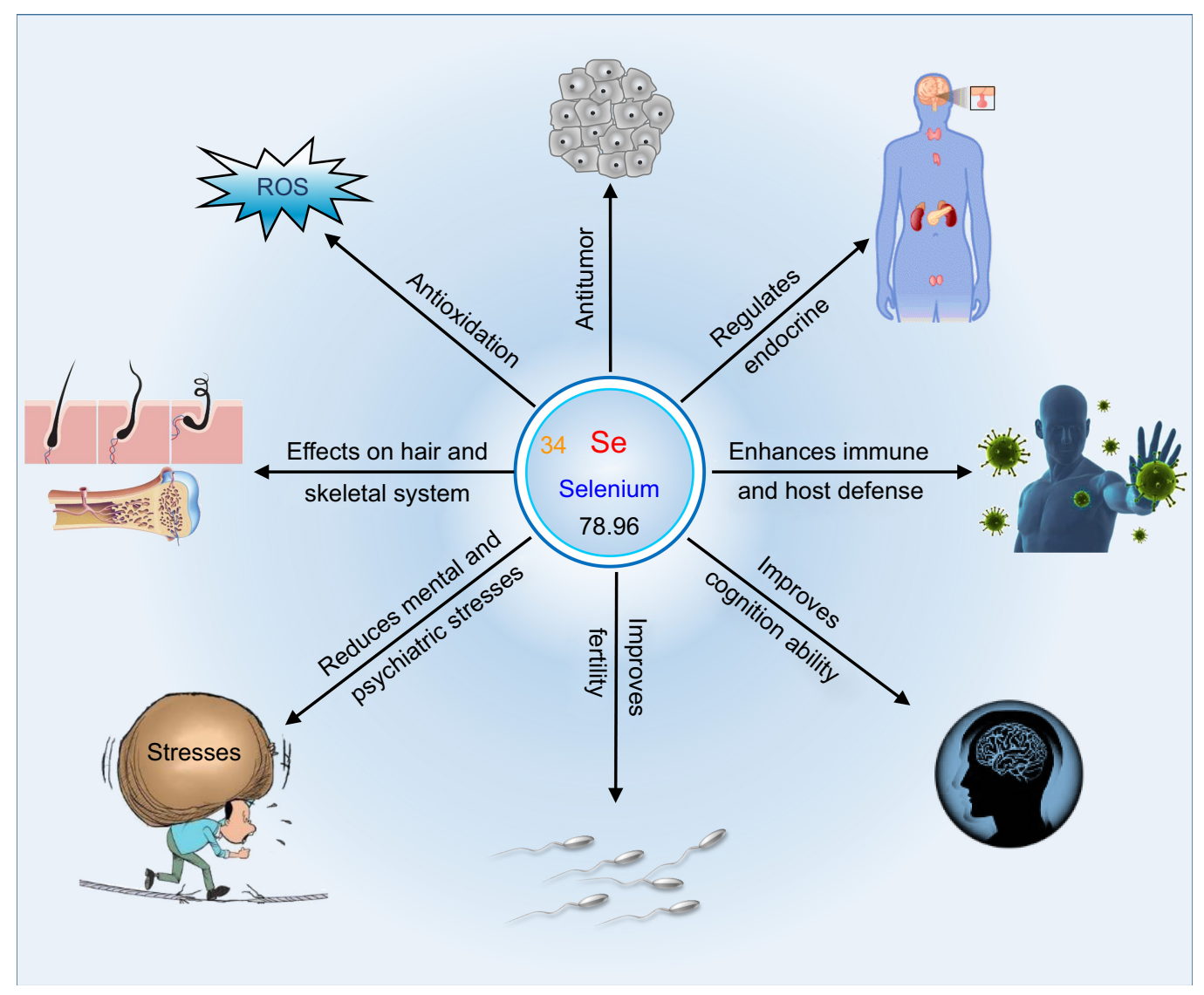

Figure I Schematic diagram outlining the principal effects of selenium on the physiological system. 
Typical examples are Sec-participated acyl transfer reactions and alkylation of selenenic acids by dimedone.

In recent years, the anticancer effects of selenium chemicals have been recognized and demonstrated in multiple experimental models. ${ }^{16}$ Tumor is a diseased status in which abnormal cells divide without control and are progressive in multiplication. Malignant tumors, known as cancer, have a strong life-threatening risk. The anticancer activity of selenium (both organic and inorganic) is supposed to be connected with its antioxidative effect, because, as yet, there has been no clinical evidence to show that selenium supplementation directly prevents the progression of cancers. Moreover, there are no selenium compounds confirmed to be effective as anticancer agents. ${ }^{17}$ Indeed, there exists an improper understanding with respect to the anticancer activity of Se. In addition to the pro-oxidant effect, the anticancer effect of selenium greatly depends on its concentration in the target site. As known, selenium has a narrow window from therapy to intoxication. In the case of selenium supplement remedy, the medications of selenium used are generally inorganic selenium compounds, such as sodium selenite and sodium selenate. Once assimilated into the body, the compounds will randomly distribute toward various tissues and organs in vivo. Hence, it is difficult to guarantee an adequate selenium concentration in the tumor tissue to produce the cytotoxic effect. Therefore, it is reasonable that common selenium compounds fail to demonstrate the desired antitumor activity, but they can act as an adjuvant to potentiate the chemotherapeutic drugs through antioxidation. To exert the antitumor effect of selenium, developing nanoscale selenium with an ameliorative in vivo pharmacokinetic distribution is a feasible strategy, which will be discussed in the special section "Se-based nanomedicines for oncotherapy" subsequently.

The effects of selenium element on the endocrine system embody in regulation of thyroxine and insulin secretion. Thyroid disease represents a medical condition that affects the function of the thyroid gland, an endocrine organ at the front of the neck that produces thyroid hormones. Selenium is a fundamental component of various selenoproteins, essential factors for the body to regulate thyroid hormones. Among identified 35 selenoproteins, three of them are involved in iodothyronine deiodinases (type I, II, and III), which play important roles in thyroid hormone metabolism. ${ }^{18}$ Indeed, the thyroid recruits the maximum level of selenium in all the organs in the body. Type I iodothyronine deiodinase (ID1) converts inactive thyroxine to its active form; type II is rich in the central nervous system, brown fat, and skeletal muscles and also participates in the activation of thyroid hormones; and type III has the function to deactivate thyroid hormones. Selenium has the priority of supply to iodothyronine deiodinases rather than GPx when its supply is inadequate. The interactions between selenium and iodine deficiencies have implications for both human health and development. ${ }^{19}$ Selenium deficiency can either exacerbate or ameliorate the effects of concurrent iodine deficiency as revealed in iodine deficiency disorders. Likewise, selenium affects insulin secretion and islet function that dominate the homeostasis of blood glucose. ${ }^{20}$ It has been shown that long-term high selenium exposure can result in potential DM risk, dysregulate insulin synthesis, secretion, and function, and affect key regulators of glycolysis, gluconeogenesis, and lipogenesis. The diabetogenic action found in healthy subjects with high selenium intake is assumed to be partly mediated by selenoproteins. However, selenoproteins can positively regulate the body glucose metabolism via the redox-sensitive signaling. Benefits and risks of selenium for DM are dialectical. A high intake of selenium in a healthy person tends to cause dysfunction of insulin secretion, whereas for a diabetic patient a high intake of selenium is beneficial to maintain the blood glucose homeostasis by its insulin-mimic function.

There is evidence that selenium can improve the body immune and host defense. ${ }^{21}$ Immune and host defense secure the body from invasion of various pathogens, from bacteria to viruses. Selenium has much potential to influence the immune system. For example, the antioxidant GPx is likely to protect neutrophils from oxygen-derived radicals that are produced to kill ingested foreign organisms. GPx is a selenium-dependent enzyme that protects cell membranes and lipid-featured organelles from peroxidative damage. It works together with vitamin E to maintain the integrity of cell membranes and participates in redox reactions with hydrogen peroxide-producing glutathione (GSH). Selenium deficiency exacerbates the redox by-product toxicity and oxidative damage to cell membranes. In addition, selenium deficiency has been proven to turn harmless viruses in hosts into virulent pathogens, ${ }^{22}$ a likely etiology in the case of Keshan disease. Periodic occurrence of new strains of influenza virus from China in Se-deficient belts provides a cue for the link between immunity and selenium deficiency.

An adequate supply of selenium is crucial to sustain various cerebral functions. In the case of low selenium status, the cerebral selenium is maintained at the expense of selenium to other cells and tissues in the body. SEPP, a circulating selenium transporter, is the most important extracellular selenoprotein that will deliver selenium to the brain and the neurons. Aaseth et $\mathrm{al}^{23}$ have amassed lots of 
data from human studies that show an association between low selenium intake (status) and various aspects of cognitive decline. They proposed the following three kinds of mechanisms accounting for cognitive improvement of Alzheimer's disease (AD) through selenium supplementation: 1) SEPP forms strong bonds with inducible metals such as copper and iron, which facilitate to block the metal-mediated aggregation of $\beta$-amyloid; 2) selenium is pivotal to prevent or inhibit the aggregation of $\tau$ proteins in the neurofibrillary tangles typical of $\mathrm{AD}$; and 3) the intracellular antioxidant role of the GPx and TrxR selenoproteins resists the development of cognitive decline. It seems clear that neurodegeneration is associated with low selenium and low SEPP levels. Therefore, it is meritorious to optimize the in vivo selenoprotein level so as to prevent neurodegeneration.

In addition to the abovementioned aspects, selenium is a key element for testosterone biosynthesis and for formation and development of normal spermatozoa. ${ }^{24}$ The high concentration of selenium in the testicular tissue is responsible for the sperm quality and male fertility. Besides, selenium plays an important role in relieving the mental/psychiatric stress. ${ }^{25}$ It shows that selenium deprivation can cause depressed mood and hostile behavior. The turnover rate of some neurotransmitters has been affected by selenium deficiency. As such, selenium is beneficial for hair growth and skeletal development. Se-dependent antioxidant enzymes prevent the damage caused to hair follicles by pollution, UV rays, and other forms of free radicals. ${ }^{26}$ Selenium also stimulates regular hair growth by helping the body to convert necessary proteins to grow hair. Both Keshan disease and muscular syndrome have been described in patients on total parenteral nutrition with insufficient selenium uptake. ${ }^{27}$ Altogether, selenium possesses multiple physiological functions and participates in many biochemical and metabolic processes within the body.

\section{Therapeutic potential of nano-selenium (nano-Se)}

Depending on multiple regulative effects, selenium is believed to support the healthy environment of the body and brings in numerous other health benefits. Upon selenium deficiency, a series of dysfunctions take place. At this time, selenium supplementation is required to remedy the morbid state due to inadequate selenium supply. In this case, selenium mostly serves as a nutrient agent, rather than a special medication. All disorders associated with selenium deficiency, such as hypothyroidism and neurodegenerative diseases, can be treated with selenium agents by peroral intake of selenate, selenite, or Se-rich foodstuff. A prerequisite is to accurately diagnose whether the disease is closely connected with Se. This can be carried out by determining the selenium level in the blood, hair, or nail and comparing it with the value in a normal person. ${ }^{28}$ Other parameters such as GPx activity and SEPP level are also good indicators of Se-deficient diseases. If identified, a corresponding measure can be carried out for the patients to restore the nutritional imbalance.

The therapeutic potential of selenium is not only reflected in its nutritive values but also embodied in its curative effects on Se-independent diseases as a factual drug. For a given therapeutic agent, the dosage form is an important factor that impacts its pharmacological effect. Ionic Se, mainly in the form of selenate and selenite, has good oral absorption and bioavailability, ${ }^{29}$ but exhibits high systemic toxicity and low bioactivity compared with organic selenium and nano-Se. This is attributable to its rapid in vivo disposition that results in high exposure to normal tissues and fast elimination from the body. Organic selenium, such as selenomethionine, which mainly exists in foods, is generally not available in large quantity. Chemical synthesis and biosynthesis have emerged as the primary sources of bioactive and bioavailable selenium. ${ }^{30}$ The current use of selenium for various medical purposes largely takes advantage of the form of nano-Se or SeNPs. SeNPs promote development of Se-based nanomedicines and establish the foundation of biomedical applications for selenium. Nanomedicine refers to the medical application of nanotechnology, involving medical nanomaterials, nanobiosensors, and biological nanodevices or robots. The utilization of nanotechnology in medicine offers some exciting possibilities whereby to more accurately diagnose, treat, and prevent various diseases. One important application of nanotechnology in medicine is to deliver active ingredients, diagnostic agents, and heat- and light inducers to specific cells or tissues using nanoparticles. At the present stage, SeNPs have been extensively investigated for their potential in treating various diseases, of which oncotherapy and antidiabetes treatment stand at the top of research hotspots. In the following sections, we will focus on the great applications of SeNPs in oncotherapy and diabetes care, along with other biomedical applications discussed.

\section{Se-based nanomedicines for oncotherapy}

Selenium acts as a potential anticancer agent with several advantages for cancer intervention, including abilities to kill cancer cells with cytotoxicity, to maintain the homeostasis of healthy cells, and to inhibit angiogenesis. Selenium is uniquely characterized by its dual effects on conditioning the pathological events: antieffect and pro-effect, depending on the chemical modality, dosage form, administration 
route, and concentration in the target site. ${ }^{17}$ For one thing, the antioxidant effect of selenium can prevent cancerization of normal cells; for another the pro-oxidant effect can also induce death of cancer cells. ${ }^{31}$ In the cancer patients, there generally exist a higher oxidative stress level and a low serum selenium level compared with a healthy person. An excess of free radicals produced in the body promotes the development of cell carcinogenesis. When a person is diagnosed with oxidative stress, selenium supplementation is favorable for cancer prevention, which is also the reason why selenium can sensitize some chemotherapeutic drugs. ${ }^{32}$ Selenium has either the antioxidant property or the pro-oxidant property, mainly relying on its concentration in the target site. ${ }^{33}$ The effects of selenium on cancer cells are closely concentration dependent, and low-to-moderate levels may stimulate growth, whereas higher levels are cytotoxic. ${ }^{34}$ This is the underlying reason that clinical trials often result in conflicting conclusions using selenium compounds as anticancer agents. Different cancer phenotypes, different chemical forms, and different target selenium concentrations will produce different curative outcomes and prognoses. It is crucial to determine the dose of selenium to treat adaptive diseases. However, the safety window of small-molecule inorganic selenium compounds largely varies according to different spectra of disorders.

Although selenium agents have great advantages in cancer treatment via multiple mechanisms, their pharmacokinetic parameters are required to be highly optimized as illustrated in Figure 2. Therefore, it is difficult to accomplish

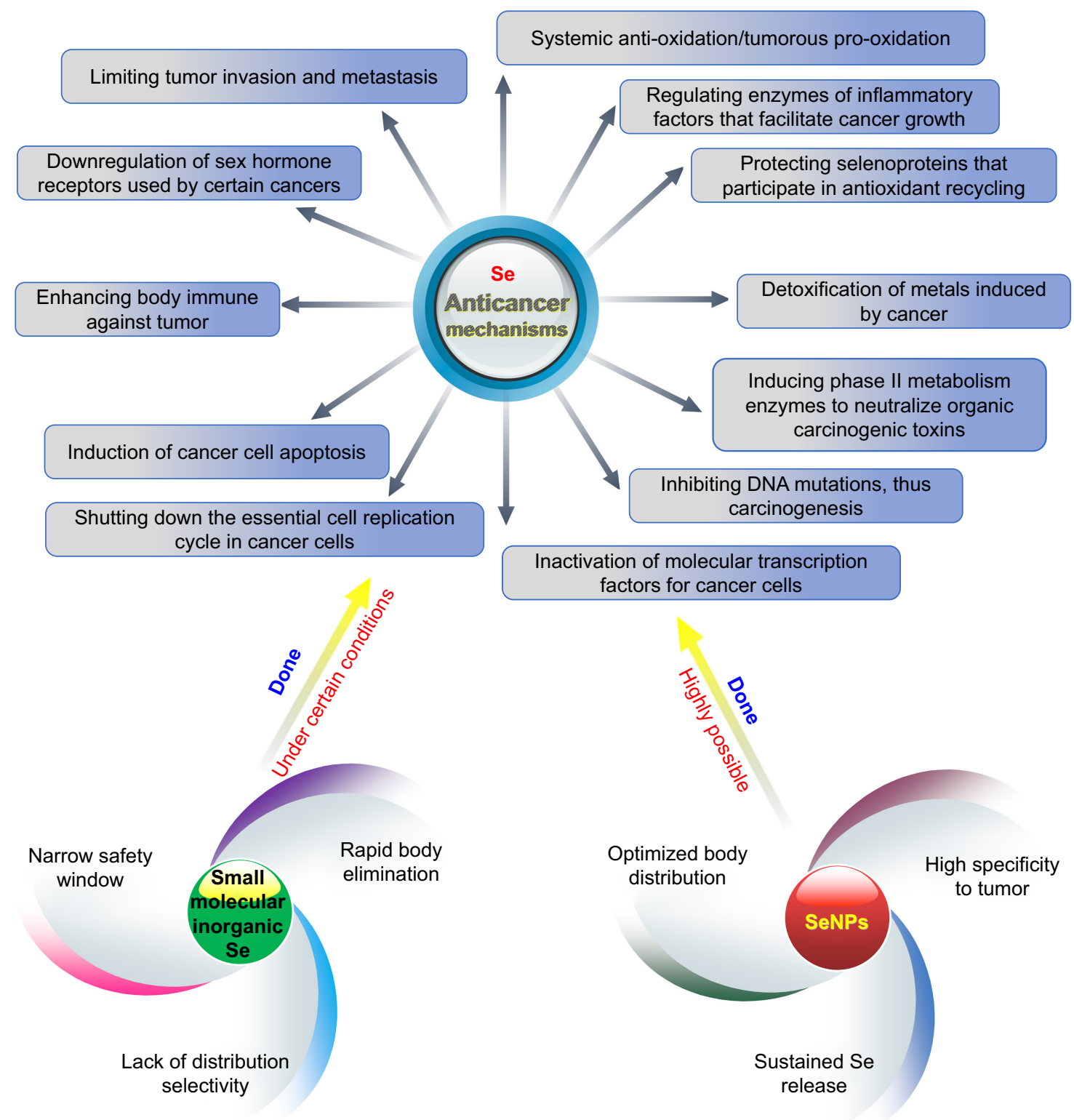

Figure 2 Anticancer mechanisms of selenium and effects of selenium modality on biological actions. Abbreviation: SeNPs, selenium nanoparticles. 
with small-molecule selenium compounds. As known, nanoparticles as drug delivery vehicles have demonstrated huge potential to improve the pharmacokinetic properties of therapeuticals. ${ }^{35}$ SeNPs are derived from inorganic selenium via a chemical process or biosynthesis, which can be used as both drug delivery carriers and therapeutic agents. ${ }^{30}$ Besides unique anticancer activity, SeNPs have been documented to possess a higher selectivity for cancerous cells to normal cells than $\mathrm{Se}^{+4}$ at similar concentrations. ${ }^{36}$ They are provided with low toxicity, high bioavailability, convenient routes of administration, and good passive targeting. Furthermore, SeNPs can sustain the release of selenium and have the aptness of targeting to tumor, thereby reducing distribution of selenium in the normal tissues and increasing accumulation in the tumorous tissues. This provides favorable conditions for implementing the precision medicine of selenium where low-to-moderate concentrations serve for body balance and high concentrations play the therapeutic role. In recent years, SeNPs have been evoking considerable interests for oncotherapy. ${ }^{37}$

Multidrug resistance represents one of the greatest challenges in cancer therapy. Liu et $\mathrm{al}^{38}$ fabricated folateconjugated SeNPs loading ruthenium polypyridyl as a novel cancer-targeting nanotherapeutic system. The nanosystem allowed direct imaging on the cellular trafficking of nanoparticles and could effectively antagonize against multidrug resistance in liver cancer. They documented that SeNPs overcoming the multidrug resistance in R-HepG2 cells were effectuated through inhibiting $\mathrm{ABC}$ family protein expression. Internalized SeNPs triggered ROS overproduction in the tumor and induced apoptosis by activating p53 and MAPK pathways. Sonkusre and Cameotra investigated the anticancer mechanism of biogenic SeNPs derived from Bacillus licheniformis JS2 in PC-3 cancer cells. ${ }^{39}$ They found that SeNPs at an Se concentration of $2 \mu \mathrm{g} / \mathrm{mL}$ were able to induce ROS-mediated necroptosis by cellular internalization. The expression of necroptosis-associated tumor necrotic factor (TNF) and interferon regulatory factor 1 (IRF1) was increased by SeNP treatment. They concluded that SeNPs induce PC-3 cell death by the ROS-mediated activation of necroptosis, independent of RIP3 and MLKL, regulated by an RIP1 kinase. Shahverdi et $\mathrm{al}^{40}$ developed folate-coated SeNPs and examined the in vitro and in vivo effects against breast cancer. Compared to SeNPs, folate-decorated SeNPs exhibited a higher cytotoxicity and a stronger tumor growth inhibiting effect in cancerous mice. Folate-modified SeNPs demonstrated a potent antiproliferative effect against 4T1 cells, significantly increased the lifespan, and prevented the tumor growth. In another study, ferulic acid-loaded SeNPs (FA-SeNPs) were synthesized via a facile and low-cost approach. ${ }^{41}$ FA-SeNPs caused the damage of tumor cells by induction of apoptosis and direct interaction with DNA. Although the antitumor effect of both single ferulic acid and SeNPs is relatively weak, the combination of the two bioactive ingredients exhibits an excellent antitumor activity. It showed that FA-SeNPs induced intracellular ROS overproduction and mitochondrial membrane potential disruption by activating caspase-3/9 to trigger HepG-2 cell apoptosis through the mitochondrial pathway. The antitumor activity of FA-SeNPs was also associated with their DNA-binding nature. However, the authors did not elucidate the synergistic effect between ferulic acid and SeNPs and how ferulic acid reinforces the antitumor effect of SeNPs. Apart from conventional SeNPs, mesoporous SeNPs coated with human serum albumin were designed and fabricated for delivery of doxorubicin (DOX) to synergistically enhance its antitumor activity. ${ }^{42}$ The mesoporous SeNPs demonstrated GSHdependent drug release, increased tumor-targeting effects, and enhanced cellular uptake through nanoparticle interaction with SPARC in MCF-7 cells. The redox-responsive SeNPs exhibited tumor-targeting abilities in tumor-bearing nude mice and not only decreased the side effects associated with DOX but also enhanced its antitumor activity.

According to the abovementioned cases, it can be acknowledged that pure SeNPs are actually valuable in oncotherapy, but the antitumor efficacy of SeNPs is still needed to be further enhanced. An applicable approach that can be implemented is to optimize the pharmacokinetics or specificity of SeNPs by surface engineering, eg, targeting modification. Some researchers have started to probe into the interest of targeted SeNP oncotherapy. ${ }^{40,41,43}$

\section{Se-based nanomedicines for diabetes care}

DM is a metabolic disorder in which the blood glucose levels are significantly higher than the normal values in the healthy people. ${ }^{44}$ Glucose comes from the diet and is regulated and controlled by insulin, a hormone that helps the glucose get into cells to supply the energy. In type $1 \mathrm{DM}$, the body does not secrete or secrete less insulin. Type 1 DM is an autoimmune condition caused by the body attacking its own pancreas with antibodies. This type of diabetes may result from a genetic predisposition, viral infection, or acute poisoning that results in organ damage. In type $2 \mathrm{DM}$, a more common type, the body does not make use of insulin well. Type 2 DM primarily arises as a result of improper diet and lack of exercise that result in insulin resistance. High blood 
glucose in the body tends to cause a series of problems, which can damage your eyes, kidneys, nerves, heart, and so on. When the body is in the prediabetic state or already diabetic, it needs medications to prevent and intervene the disease progression.

The association between serum selenium level and DM remains confused according to previous reports. The surveys on the interrelation of selenium and DM often turn up with contradictory conclusions. One prospective study from France found that old men with higher selenium levels had a lower risk of developing type $2 \mathrm{DM}$ or impaired fasting glucose later, but no association with women..$^{45} \mathrm{~A}$ long-term study revealed that higher selenium level in the toenail was associated with a lower risk of type $2 \mathrm{DM}$ in US adults at the dietary level of intake. ${ }^{46}$ A survey of Spanish adults showed an association between selenium level and type 2 $\mathrm{DM}$ as well as an interaction with genes. ${ }^{47} \mathrm{~A}$ study specific to Chinese seniors discovered that higher selenium level was connected with a higher risk of type $2 \mathrm{DM} .{ }^{48}$ Therefore, there is an intrinsic correlation between selenium and DM. High selenium intake is not proposed for healthy people, since this may increase the risk of developing DM owing to the pro-oxidant effect of Se. However, as far as diabetic patients are concerned, it is a proper option to remedy the metabolic disturbance through the intake of an appropriate amount of selenium. Diabetic patients are frequently subjected to inflammatory response and oxidative stress due to undue exposure to high blood glucose. ${ }^{49}$ Selenium has been reported to have excellent antioxidant and anti-inflammatory actions. ${ }^{50,51}$ It has been reported that selenium concentration was higher in diabetic patients than in healthy people. ${ }^{52,53}$ Once developing DM, the body needs more antioxidant species to fight against oxidative and inflammatory responses. Therefore, selenium as a potent antioxidant and a physiological modulator is inspired for use in DM care. In this respect, SeNPs (nano-Se) possess many therapeutic advantages over small-molecule selenium and have been actively investigated for their antidiabetic efficacy.

Oxidative stress involved in endothelial dysfunction mediates the onset and progression of diabetes. Brownlee ${ }^{54}$ has proposed a molecular mechanism on the diabetic vascular injury that is hyperglycemia-induced ROS overproduction by the mitochondrial electron transport chain. SeNPs not only have the function of counteracting oxidative stress but also possess an intrinsic hypoglycemic activity and can be used as a hypoglycemic agent alone. Therefore, both type 1 and type 2 DM can be treated with SeNPs through attenuating oxidative damage and sensitizing insulin.
Al-Quraishy et $\mathrm{al}^{55}$ investigated the hypoglycemic effect of SeNPs in streptozotocin (STZ)-induced diabetic rats, a type 1 DM model. The diabetic rats exhibited significantly decreased fasting blood glucose levels after treatment with SeNPs for 28 days. The serum insulin concentration was also enhanced by SeNPs. SeNPs were shown to be able to reduce hepatic and renal function markers, total lipids, total cholesterols, triglyceride and low-density lipoprotein cholesterol levels, and glucose-6-phosphatase activity, but as opposed to malic enzyme, hexokinase and glucose-6-phosphate dehydrogenase activity, liver and kidney glycogen contents, and high-density lipoprotein cholesterol levels. In addition, SeNPs prevented the histological injury in the hepatic and renal tissues of rats. The study indicates that SeNPs can alleviate hyperglycemia and hyperlipidemia in diabetic subjects, possibly by eliciting an insulin-mimetic effect. Liu et al ${ }^{56}$ prepared SeNPs by introducing catathelasma ventricosum polysaccharides (CVPs) to the redox system of selenite and ascorbic acid and investigated their antidiabetic activity in STZ-induced diabetic mice. CVPs-SeNPs exhibited a potential antidiabetic effect as revealed by serum profiles of glucose and antioxidant enzyme levels. Moreover, CVPs-SeNPs had significantly higher antidiabetic activity than other selenium preparations $(P<0.05)$, such as SeNPs, Sec, and sodium selenite. At the same time, the research group fabricated chitosan-stabilized SeNPs (CTS-SeNPs) through reduction of selenite acid with ascorbic acid in a chitosan solution. ${ }^{57}$ They pointed out that CTS-SeNPs, at a selenium dose of $2.0 \mathrm{mg} / \mathrm{kg}$, could produce higher antidiabetic activity than other doses of SeNPs and other selenium agents with the same selenium dose. A preclinical study on the antidiabetic potential of SeNPs was carried out by Ahmed et al. ${ }^{58}$ In contrast, in this study, SeNPs were orally delivered to diabetic rats in the form of liposomes instead of original SeNPs. The STZ-induced rats were treated with liposomal SeNPs for 21 days and posed significant decline in serum glucose, pancreatic peroxides, and inflammatory mediators, and significant increase in serum insulin and pancreatic antioxidants as well as significant improvement in the immunohistochemical indices of insulin and glucagon. The study provided sound evidence in support of the antidiabetic potency of SeNPs. Liposomal SeNPs preserved the pancreatic $\beta$ cell integrity with consequent increment of insulin secretion and thus resulted in glucose depletion, repression of oxidative stress, potentiation of the antioxidant defense system, and inhibition of pancreatic inflammation. These findings indicate that SeNPs can strikingly enhance the therapeutic index of selenium. Se-based nanomedicines have shown great importance of application to diabetes care. 


\section{SeNPs for other biomedical purposes}

Aside from oncotherapy and antidiabetes treatment, the therapeutic potential of SeNPs has been profoundly unearthed in the past decade. ${ }^{59}$ Depending on multiple bioactivities and physiological regulating effects, SeNPs are extensively utilized to deal with oxidative stress injury, inflammatory diseases, Se-related thyroid dysfunction, AD, bacterial/fungal infection, viral infection, chemo/radiotherapy adjuvants, deintoxication, fertility enhancement, medical diagnosis, etc. Figure 3 generalizes the major biomedical applications of SeNPs beyond anticancer and antidiabetes effects. The performance and suitability of SeNPs as pleiotropic nanomaterials have been verified with prominent excellences over small-molecule selenium compounds.

Zhu et al ${ }^{60}$ developed Ulva lactuca polysaccharidedecorated SeNPs and investigated their anti-inflammatory efficacy in an acute colitis model. Low selenium status exacerbates inflammatory bowel disease progression that involves hyperinflammation in the digestive tract. SeNPs resulted in a significant protective effect on the acute colitis. SeNP treatment mitigated the body weight loss and colonic inflammatory damage in mice and ameliorated macrophage infiltration. The anti-inflammatory effects of SeNPs were shown to be relevant to modulation of cytokines including IL- 6 and TNF- $\alpha$ through inhibiting the activation of macrophages by suppressing the nuclear translocation of NF- $\mathrm{\kappa B}$ responsible for the transcription of these pro-inflammatory cytokines. To evaluate the anti-inflammatory effect of SeNPs on mice jejunum infection, Alkhudhayri et $\mathrm{a}^{6}{ }^{61}$ established the mice infection model with sporulated oocysts of Eimeria papillata and compared the anti-inflammatory activity of SeNPs with sodium selenite. SeNPs were found to be able to regulate the gene expression of mucin 2, IL-1 $\beta$, IL-6, interferon- $\gamma$, and tumor necrosis factor $\alpha$ in the jejunum. It shows that SeNPs are more effective than sodium selenite with reference to their anticoccidial, antioxidant, and antiinflammatory roles against eimeriosis-induced jejunum infection. The zero-valent selenium has low toxicity and excellent bioavailability, but it is unstable and easy to change into an inactive form. To stabilize zero-valent selenium and explore its antioxidant capacity, Zhai et a ${ }^{62}$ prepared SeNPs with chitosan as a stabilizer. The antioxidant tests on 2,2-diphenyl-1picrylhydrazyl (DPPH), 2-2'-azino-di-(3-ethylbenzthiazoline sulfonate) (ABTS), and lipid peroxide models showed that the synthesized SeNPs could scavenge free radicals at different levels. SeNPs inhibited the production of ROS in a selenium concentration-dependent mode at the cell level. Following administration both via the topical route and the oral route, SeNPs efficiently protected GPx activity and prevented the lipofuscin formation induced by UV radiation or D-galactose in mice. However, the acute toxicity of optimal SeNPs was ten times lower than that of sodium selenite. In an in vivo study, rats with arthritis showed depletion of superoxide dismutase (SOD), catalase (CAT), and GPx in the liver, kidney, and spleen as well as an increase in serum C-reactive protein. These disturbances were reversed by oral

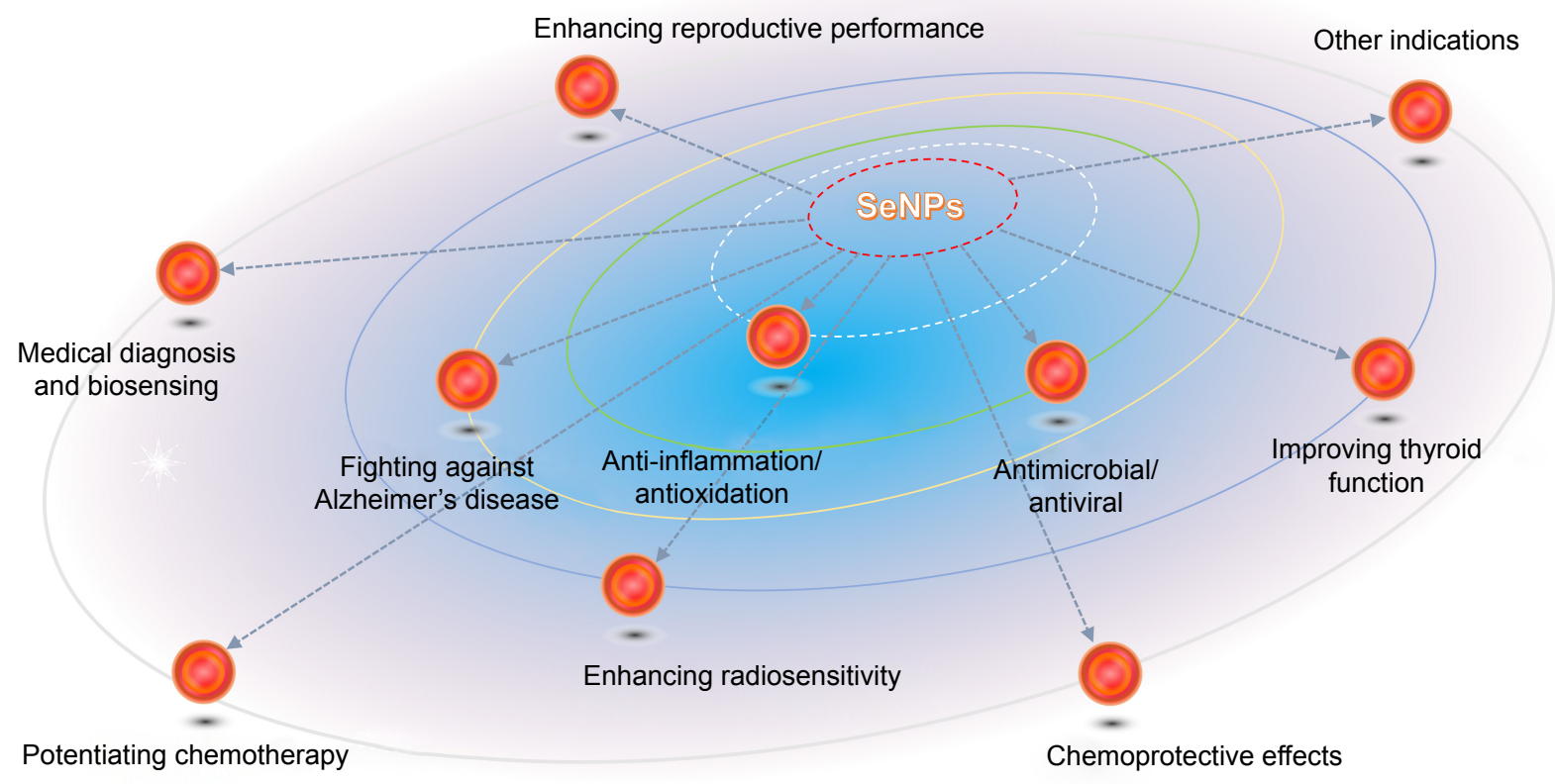

Figure 3 Biomedical applications of SeNPs for regulation of physiological functions and treatment of various diseases. Abbreviation: SeNPs, selenium nanoparticles. 
administration of SeNPs with different doses of selenium. ${ }^{63}$ All the abovementioned facts provide evidence that SeNPs have good anti-inflammatory and antioxidant effects that can be applied to inflammatory and oxidative damages occurring within the body.

The antibacterial, antifungal, and antiviral activities of SeNPs are the important features of SeNPs. Srivastava and Mukhopadhyay ${ }^{64}$ synthesized SeNPs by means of a green approach using non-pathogenic, economic, and easy to handle bacterium Ralstonia eutropha and assessed their antimicrobial property. The biologically synthesized SeNPs showed excellent antimicrobial activity, which inhibited 99\% growth of Pseudomonas aeruginosa, Staphylococcus aureus, Escherichia coli, and Streptococcus pyogenes at a concentration of $100,100,250$, and $100 \mu \mathrm{g} / \mathrm{mL}$ of SeNPs, respectively. In addition, it was found that SeNPs at $500 \mu \mathrm{g} / \mathrm{mL}$ inhibited the growth of pathogenic fungi Aspergillus clavatus. The antimicrobial efficacy of SeNPs was comparable with commercially available antibiotic drug ampicillin. With respect to antifungal pathogens, Lara et $\mathrm{al}^{65}$ synthesized SeNPs through an irradiating selenium pellet technique by nanosecond pulsed laser ablation in a solution with chitosan as a capping agent. The synergistic fungicidal effect of SeNPs and chitosan was evaluated by the combination index theorem of Chou-Talalay. The nanobiomaterial showed a potent fungicidal effect against a preformed Candida albicans biofilm in a dose-response mode. Chitosan facilitated adhesion and penetration of SeNPs, thereby resulting in disruption and distortion of fungus cell walls. Furthermore, the antiviral capacity of SeNPs has been tested on H1N1 influenza virus. Li et $\mathrm{a}^{66,67}$ constructed oseltamivir and amantadine-loaded SeNPs and investigated their antiviral activities against H1N1 influenza virus, respectively. ${ }^{66,67}$ The combined use of antiviral agent and SeNPs collectively inhibited the generation of ROS and activation of p53 phosphorylation and $\mathrm{AKT}$, demonstrating to be more potent against H1N1 than a single antiviral agent.

Besides iodine, the well-functioning of the thyroid gland also requires participation of a number of elements, including Se, iron, zinc, copper, and calcium. ${ }^{68}$ Selenium is accounted to be the key element for homeostasis of thyroid hormones and thus has important significance of treating Se-deficit thyroid dysfunction. Atteia et $\mathrm{al}^{69}$ investigated and characterized the potential protective mechanism of SeNPs against lead acetate $(\mathrm{PbA})$-induced thyrotoxicity. It was found that a 15 -week treatment with SeNPs $(0.5 \mathrm{mg} / \mathrm{kg}$, intraperitoneal [ip]) significantly alleviated the hormone indices of decreased free triiodothyronine (fT3) and thyroxine (fT4) levels as well as fT3/fT4 ratio and increased thyroid-stimulating hormone (TSH) levels. SeNP treatment also resulted in reduced accumulation of plumbum $(\mathrm{Pb})$ in the serum and thyroid tissues and proper maintenance of thyroidal pro-oxidant/antioxidant balance and ID1, an essential enzyme for converting T4 into active T3. In addition, the expression of ID1 mRNA (miR$224)$ in the thyroid tissues was significantly downregulated in SeNPs and PbA co-treated rats. The results indicate that SeNPs can prevent $\mathrm{PbA}$-induced hypothyroidism and oxidative damage in the thyroid tissues by modulating selenoenzymes and suppressing miR-224, a direct complementary target of ID1.

Amyloid $\beta(A \beta)$ plaque accumulation and oxidative stress in the brain have important implications in the etiology of AD. In the past decade, it has motivated a great interest in integrating selenium and various antioxidant agents into nanoparticles to treat AD. ${ }^{70}$ Yin et $\mathrm{al}^{71}$ developed sialic acid (SA)-modified SeNPs coated with a high blood-brain barrier (BBB) permeability peptide-B6 peptide (B6-SA-SeNPs) for potential use in AD. B6-SA-SeNPs as a synthetic selenoprotein analog showed high permeability across the BBB and could serve as a novel nanomedicine for $\mathrm{AD}$ intervention. The inhibitory effects of B6-SA-SeNPs on A $\beta$ peptide fibrillation have been demonstrated in PC12 cells and bEnd 3 cells. B6-SA-SeNPs not only effectively inhibited $A \beta$ aggregation but also disaggregated preformed $A \beta$ fibrils into non-toxic amorphous oligomers. These findings suggest that B6-SASeNPs may provide a promising option for AD treatment through overcoming BBB and anti-amyloid and antioxidant mechanisms.

Se supplementation can bring about additional benefits to radiotherapy for patients with low selenium status. Moreover, selenium agents are clinically encouraged to be used as adjuvants for radioprotection in tumor radiotherapy. ${ }^{72}$ ${ }^{125}$ I seeds demonstrate higher efficacy and fewer side effects than traditional X-ray radiotherapy, but they possess radioresistance in clinical applications. Hence, design and synthesis of sensitizers that can enhance the sensitivity of cancer cells to ${ }^{125} \mathrm{I}$ seeds are of great significance for radiotherapy. To achieve synergetic chemoradiotherapy, Chan et $\mathrm{al}^{73}$ utilized cancer-targeted SeNPs to sensitize cancer cells subjected to continuous $\gamma$ radiation. Cancer-targeted SeNPs, based on the Auger electron and Compton effects of selenium atoms, in combination with ${ }^{125}$ I radiation synergistically inhibited cancer cell growth and colony formation through inducing cell apoptosis and cell cycle arrest. In another report, Yang et $\mathrm{al}^{74}$ constructed folic acid-conjugated SeNPs (FA@SeNPs) as a cancer-targeting and radiosensitivity agent to enhance 
the radioactivity of ${ }^{125} \mathrm{I}$ seeds to potentiate the anticancer efficacy. Compared with X-ray irradiation, ${ }^{125} \mathrm{I}$ seeds exhibited a good synergistic effect with FA@SeNPs, drastically increasing ROS overproduction and influencing the cell cycle distribution. The underlying mechanisms of enhanced ${ }^{125} \mathrm{I}$ seeds radiotherapy in combination with SeNPs were demonstrated to be associated with induction of p53-mediated DNA damage and activation of mitogen-activated protein kinase (MAPK). Besides ameliorative in vivo antitumor activity, this combined therapy also showed lower systemic toxicity relative to conventional X-ray radiotherapy. These studies manifest that SeNPs can be used as an effective radiosensitivity agent to enhance the clinical efficacy of cancer radiotherapy.

In addition to application in radiotherapy, SeNPs have also been used to increase the sensitivity of chemotherapy by co-administration with a chemotherapeutic drug. AbdRabou et $\mathrm{al}^{75}$ investigated whether SeNPs could induce the chemosensitivity of 5-fluorouracil (FU)-loaded nanoparticles (FU-NPs) in breast and colon cancer cell lines. They found that SeNPs potentiated the chemosensitivity of FU-NPs in MCF7 and Caco-2 higher than MDA-MB-231 and HCT 116 cancerous cell lines, which was fulfilled by inhibiting cancer cell bioenergetics via glucose uptake blockage. FUNPs increased the levels of NO and MDA in media over cancer cells, whereas FU-NPs together with SeNPs rebalanced the redox status. MCF7 cell line was more sensitive to the combination of FU-NPs and SeNPs. This may be because that SeNPs have the aptness of selectively entering into cancerous tissues more than into normal cells, which broadens the chemotherapeutical window via enhancing the immunomodulation to normal cells and killing cancerous cells. ${ }^{76}$ In another work, SeNPs were concurrently used with irinotecan to enhance anticancer efficiency and reduce systemic toxicity. ${ }^{77}$ The combination of SeNPs and irinotecan dramatically inhibited tumor growth and significantly induced tumor cell apoptosis in the HCT-8 cell xenograft model. Also, the combination of SeNPs and DOX showed better anticancer effect than individual treatments. ${ }^{78}$ These results suggest that SeNP is a promising adjuvant to potentiate the chemotherapeutic effect.

High exposure to toxic species, including cytotoxic agents, heavy metals, and harmful ions, becomes a great concern for human health. SeNPs can be used as counterpoisons by virtue of excellent poison resistance. Cyclophosphamide (CP) is one of the representative anticancer drugs but has serious deleterious effects on normal host cells due to its non-specific action. Co-administration of CP and SeNPs caused a significant reduction in tumor volume, packed cell volume, and viable tumor cell count, whereas increased survivability of the tumor-bearing hosts. ${ }^{79}$ The chemoprotective and chemosensitizing properties of SeNPs have been confirmed in tumor-bearing mice with attenuation of $\mathrm{CP}$-evoked toxicity and amplification of chemotherapeutic effect. Cisplatin as an anticancer alkylating agent is broadly used for cancer treatment. Despite low cost and vast use in clinic, cisplatin possesses a number of side effects. It can induce DNA adducts and form DNA cross-links that interfere with cellular metabolism, such as DNA replication and transcription, thus triggering cell death. Li et al ${ }^{80}$ prepared 11-mercapto-1-undecanol-functionalized SeNPs (Se@MUN) and investigated their ability to reverse cisplatin-induced nephrotoxicity. Se@MUN exhibited free radical scavenging activity and significantly attenuated cisplatin-induced nephrotoxicity and overproduction of intracellular ROS. Likewise, Rezvanfar et al ${ }^{81}$ demonstrated that SeNPs could prevent cisplatin-induced gonadotoxicity by an antioxidant mechanism. Co-administration of SeNPs with cisplatin significantly improved the serum testosterone, sperm quality, and spermatogenesis and decreased cisplatin-induced free radical toxic stress and spermatic DNA damage. The chemoprotective effects of SeNPs on neurotoxicity and nephrotoxicity caused by subchronic cadmium chloride exposure were verified by Sadek et al. ${ }^{82}$ They stated that SeNPs ameliorated the adverse neurological and nephrotoxic effects partially through scavenging of free radicals, metal ion chelation, averting apoptosis, and altering the cell-protective pathways. In another study, the protective effect of SeNPs against 7,12-dimethylbenz(a)anthracene (DMBA)-induced immunotoxicity was examined. ${ }^{83}$ The survival of leucocytes in the SeNP group was three times as high as that of the control group and four times in terms of phagocytes. Moreover, the recovery in the bone marrow and the regenerative capacity of granulopoiesis were significantly improved. SeNPs have also been reported to minimize the risk of iron overabundance. ${ }^{84}$ The chemoprotective effects of SeNPs against various heavy metal-induced toxicities have been explored and favorable results were yielded, including arsenite-induced chronic toxicity, ${ }^{85}$ hexavalent chromium-induced thyrotoxicity, ${ }^{86}$ and cadmium-induced nephrotoxicity. ${ }^{87}$

Applications of SeNPs for medical diagnosis and biosensing depend on the physiochemical properties of element selenium. The facile conjugation makes selenium a useful element for the identification of biological components. SeNPs with different sizes and area densities were separately fabricated by controlling the rapid adsorption 
of molecule-imprinted nanocomposites and photocatalytic reaction of $\mathrm{TiO}_{2}$ nanoparticles. ${ }^{88}$ Thanks to the biological activity, SeNPs were tentatively used for dot-blot immunoassays with multiple native antigens for rapid serodiagnosis of human lung cancer. The sensitivity of dot immunoassays for the detection of progastrin-releasing peptide (ProGRP) is as low as $75 \mathrm{pg} / \mathrm{mL}$ and the detection time is only 5 minutes. No positive results accompany with other commonly potential interfering substances, such as carcinoembryonic antigen and BSA. The intrinsic fluorescence of SeNPs and their imaging capability have been checked by Khalid et al. ${ }^{89}$ They identified the intrinsic fluorescence of SeNPs and found that it was highly beneficial for nanoscale imaging of biological structures. The photoluminescence of SeNPs spectra covers from visible to near infrared area, which is valuable for in vitro imaging and tracking in fibroblast cells. The technique overcomes limitations of commonly used approaches of imaging with tagged fluorescent proteins and dyes and averts potential adverse cellular effects or phototoxicity caused by the additives. Furthermore, SeNPs have been explored as a peroxide biosensor. For example, Wang et al ${ }^{90}$ synthesized semiconductor monoclinic SeNPs through a biologically modified method for precise detection of $\mathrm{H}_{2} \mathrm{O}_{2}$. It showed that the $\mathrm{H}_{2} \mathrm{O}_{2}$ biosensor had high sensitivity and affinity for $\mathrm{H}_{2} \mathrm{O}_{2}$ with a detection limit of $8 \times 10^{-8} \mathrm{M}$. Excess exposure to peroxides poses significant impairment to the central nervous system of human beings. SeNPs may serve for the diagnosis and evaluation of the in vivo oxidative stress status.

The effect of SeNPs on the reproductive performance is a great interest worthy of investigation. Selenium is essential for testosterone biosynthesis and formation and development of normal spermatozoa. ${ }^{91}$ In the testicular tissue, there is a high concentration of selenium responsible for sperm quality and male fertility health. Liu et $\mathrm{al}^{92}$ investigated the effect of SeNPs on the reproductive performance of male Sprague Dawley rats at supranutritional and non-lethal levels. After oral gavage for 2 weeks, the supranutritional doses $(0.2,0.4$, or $0.8 \mathrm{mg} \mathrm{Se} / \mathrm{kg})$ promoted the sperm motility $(P<0.001)$ and movement parameters $(P<0.05)$, and the content of testosterone in the serum was increased by SeNPs treatment as well. No significant effects were observed on antioxidant enzyme activities and mRNA level of GPx at supranutritional doses. SeNPs produce a positive effect on the reproductive function at a fitting dose, which may be promising for the treatment of Se-deficit infertility.

Se-based nanomedicines provide attractive characteristics with various possible biomedical applications. Apart from the abovementioned reports, SeNPs have been explored for promoting hair follicle development and fetal growth ${ }^{93}$ and treating Kashin-Beck disease, ${ }^{94}$ Leishmania major,${ }^{95}$ hydatid cysts, ${ }^{96}$ hepatic damage, ${ }^{97}$ and diabetic nephropathy. ${ }^{98}$

\section{SeNPs serving for drug delivery}

SeNPs are customizable drug delivery nanocarriers that show good bioavailability, higher delivery efficacy, and lower toxicity than free selenium. The well tunable and multivalent surface of SeNPs enables them to effectively load chemical drugs or biomacromolecules by covalent or non-covalent bonds. On the surface of SeNPs, there are residual unreacted ions or naked stabilizers. These species are generally positively or negatively charged that can conjugate with $\mathrm{NH}, \mathrm{C}=\mathrm{O}, \mathrm{COO}^{-}$, and $\mathrm{C}-\mathrm{N}$ groups of active pharmaceutical ingredient (API), which imparts SeNPs superior adsorptive capacity to load, stabilize, and deliver drug. ${ }^{99}$ In general, Se-based nanoparticles can be manufactured through the following two approaches: 1) in situ formation via reduction ${ }^{100}$ and 2) fabrication on the base of nanoparticles, ${ }^{101}$ as illustrated in Figure 4. In the former, SeNPs are synthesized from different precursor materials, reducing agents, and stabilizers through a chemical reaction. The precursors contain selenium compounds, such as sodium selenite, sodium selenate, and selenic acid. Reducing agents can utilize ascorbic acid, mercaptoethanol, and GSH. Stabilizers not only facilitate the formation of physically stable colloidal SeNPs but also confer SeNPs a functionality, rendering the inorganic nanoparticles suitable for drug delivery. Polysaccharides, proteins, and polymers are involved in the fabrication of SeNPs, which modulate the physicochemical properties and bioavailability of SeNPs. In the latter, selenium is attached onto the surface of prefabricated nanoparticles and forms Se-plated nanoparticles by capping. It is crucial to control the size of SeNPs and select a suitable functional modifier for the first strategy so as to obtain satisfactory drug loading and pharmacokinetic property. The second strategy has a great flexibility for drug loading, since the payload has already been encapsulated into nanoparticles. The accessibility in engineering has sparked intense interest of using SeNPs as a nanovehicle of therapeutic agents to achieve a synergistic effect between selenium and its therapeutic cargo. SeNPs can also be modified with functional ligands to achieve specific cell or organelle targeting, such as targeting to cancerous cells and the mitochondria of diverse cells. Nanotechnology-inspired nanomedicines intended for mitochondrial dysfunction-related diseases have become a major arena of interest in precision therapy. ${ }^{102}$ Many diseases are associated with mitochondrial dysfunction, including cancer, cardiovascular diseases, diabetes, 

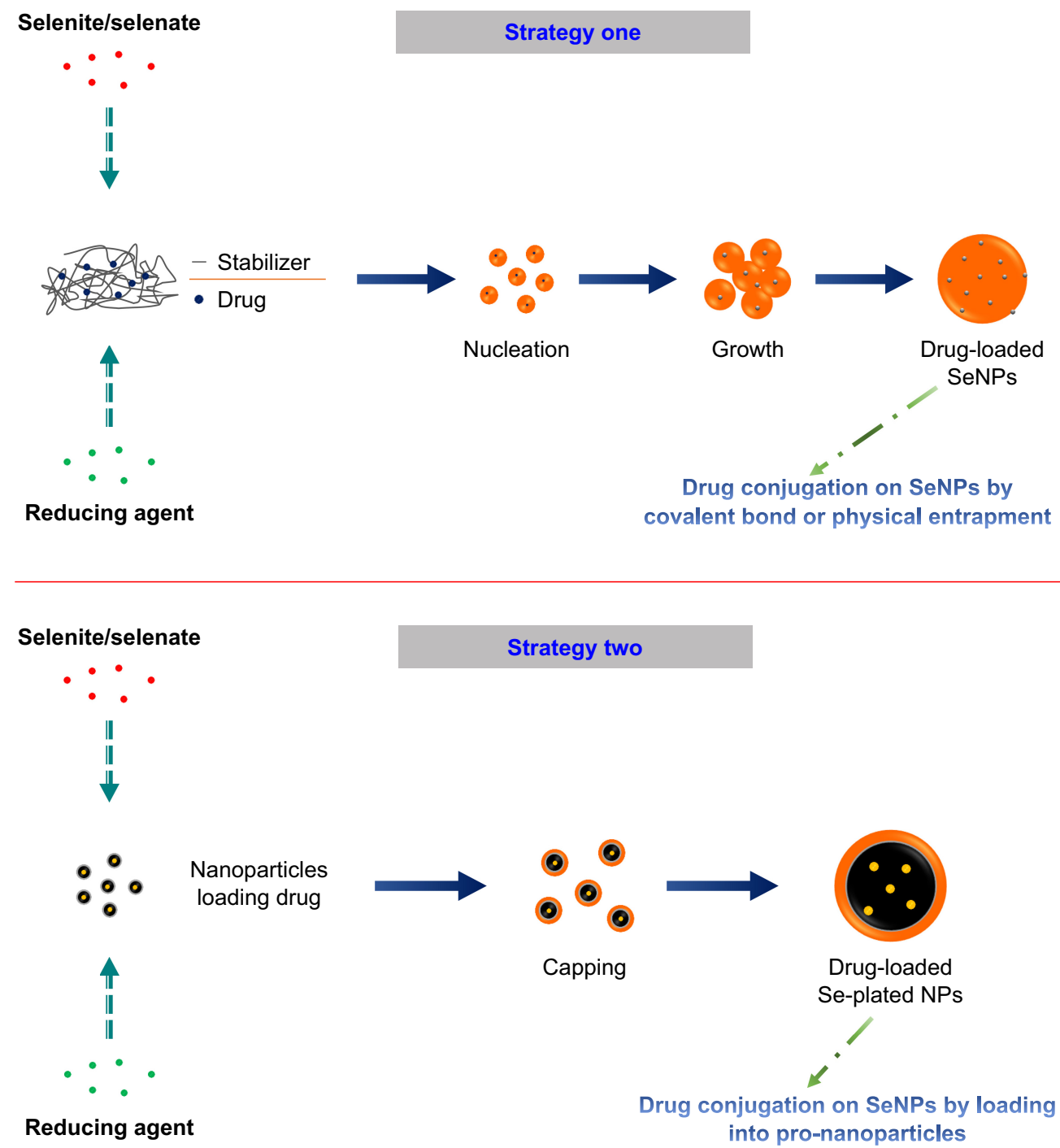

Figure 4 Preparative techniques of SeNPs and Se-functionalized nanoparticles that are used for drug delivery.

Abbreviation: SeNPs, selenium nanoparticles.

and neurological disorders. Certain organic cations can penetrate the mitochondrial membrane and can be employed to deliver therapeuticals into the mitochondria, such as chitosan ${ }^{103}$ and triphenylphosphonium. ${ }^{104}$ These cationic compounds can be utilized to fabricate SeNPs through covalent conjugation for targeted drug delivery.

\section{Anticancer drugs}

In virtue of intrinsic anticancer activity, SeNPs are most widely used for systemic delivery of various anticancer drugs. Bioactivity coupled with a higher selectivity to cancer cells promises a targeted delivery with reduced systemic toxicity and enhanced chemotherapeutic efficacy. ${ }^{36}$ To take advantage of both facets, Kumari et a ${ }^{105}$ expropriated the tumoricidal characteristics of SeNPs and designed curcumin-loaded SeNPs (Cur-SeNPs) to achieve an enhanced therapeutic effect. The in vitro results showed that Cur-SeNPs were most effective on colorectal carcinoma cells (HCT116) and possessed pleiotropic anticancer effects primarily ascribable to an elevated level of autophagy and apoptosis, which could be deduced from significant upregulation of autophagy-associated and pro-apoptotic proteins, downregulation of antiapoptotic proteins, and cytochrome $\mathrm{C}$ release from mitochondria along with reduced $\mathrm{NF} \kappa \mathrm{B}$ signaling and EMT-based machineries. The in vivo studies on the Ehrlich's ascites carcinoma (EAC) mice model indicated that Cur-SeNPs significantly reduced the tumor progression and enhanced the mean survival time of tumor-bearing EAC mice. Paclitaxel (PTX) represents the best natural anticancer drug that has been discovered. Bidkar et al ${ }^{106}$ developed SeNPs for the delivery of PTX and assessed their antiproliferative efficacy against cancer cells in vitro with potential 
mechanistic insight. PTX-loaded SeNPs gave rise to significant antiproliferative activity against cancer cells by inducing apoptosis caused by G2/M phase arrest. Efficient induction of apoptosis in various cancer cells through PTX-loaded SeNPs might lead to their potential application in oncotherapy. In order to enhance the anticancer efficacy of oridonin, GE11 peptide-conjugated SeNPs (GE11-SeNPs) targeting EGFRoverexpressed cancer cells were synthesized. ${ }^{107}$ It was found that GE11-SeNPs raised the cellular uptake of oridonin in cancer cells, resulting in enhanced cancer inhibition against cancerous cells and reduced toxicity against normal cells. In a xenograft model of nude mice, oridonin-loaded GE11-SeNPs significantly inhibited the tumor growth by suppressing the tumor angiogenesis and activating the immune system. This tumor-targeted, oridonin-loaded SeNP provides a new strategy for synergistic treatment of cancer with higher efficacy and reduced side effects. Targeted co-delivery of epirubicin (EPI, an anticancer agent) and NAS-24 aptamer (an apoptosis inducer) to cancer cells using SeNPs for enhanced tumor response in vitro and in vivo has been carried out by Jalalian et al. ${ }^{108}$ In this study, 5TR1 aptamer-functionalized EPI-loaded SeNPs were fabricated and significantly reduced the toxicity in non-target cells and inhibited the tumor growth in cancer-bearing mice compared to EPI treatment alone. As an interesting example, in our group, we successfully constructed Se-functionalized liposomes (SeLPs) for systemic delivery of Dox by in situ selenium coating to liposomes. ${ }^{109}$ It was shown that Dox-loaded SeLPs possessed a sustained release effect for Dox and could increase the cellular uptake of Dox compared with the performance of conventional liposomes. Selenium coating strengthens the structure of liposomes and preserves liposomes from swift collapse while circulating in the body and thus postpones the overall release of drug in vivo. In addition, selenium attached to liposomes doubles the anticancer effect of liposomal Dox by offering its potency to the cargo. Therefore, the designed SeLPs prolong the circulation time of Dox and enhance the anticancer effect via the synergy between Dox and selenium. Anyhow, delivering anticancer drugs via SeNPs will develop into a major arena of Se-based nanomedicines.

\section{Protein and peptide drugs}

In recent years, SeNPs have unfolded important applications in protein and peptide delivery. Proteins as a kind of stabilizer are oftentimes involved in the preparation of SeNPs. ${ }^{110,111}$ Based on the principle of multivalent selenium able to couple with endogenous proteins, ${ }^{112}$ protein and peptide drugs can be formulated with SeNPs and achieve an efficient delivery.
The inaugural survey of SeNPs for protein drug delivery was proposed by our group involving oral delivery of insulin through SeNPs. ${ }^{113}$ In this case, insulin-loaded SeNPs (INSSeNPs) were fabricated by an ionic cross-linking/in situ reduction technique as illustrated in Figure 5. Insulin acts as both a therapeutic agent and a stabilizer of SeNPs, which is directly encapsulated within SeNPs by entanglement and thus can circumvent gastrointestinal degradation from digestive enzymes. The resultant INS-SeNPs possess a small particle size, high drug entrapment rate, controllable insulin release, and prominent physiological stability in the digestive fluids. INS-SeNPs produced a significant hypoglycemic effect in both normal and type 2 diabetic rats (GotoKakizaki, GK) after oral administration. The relative pharmacological bioavailability was up to $9.15 \%$ compared with subcutaneously injected insulin. Meanwhile, the blood insulin levels were markedly elevated by SeNPs. Ex vivo intestinal imaging and cell tests provided evidence on excellent intestinal permeability of INS-SeNPs. Mechanically, INS-SeNPs could alleviate oxidative stress, improve pancreatic islet function, and promote glucose utilization. This study provides proof of concept for the use of SeNPs to deliver protein drugs and synergize them for treatment. However, over and above insulin, we have not caught sight of any other protein drugs being delivered with SeNPs. The interactional nature of selenium and protein that couple with each other and form SeNPs is still needed to be further explored.

SeNPs applied to peptide drug delivery annotate another important trait thereof. Peptides constitute the basic structure of proteins and thus can conjugate with SeNPs similar to proteins. BAY 55-9837 is a polypeptide showing a vasoactive intestinal peptide receptor 2 (VPAC2) receptor antagonism with potential activity for type $2 \mathrm{DM}$, but is limited for use due to poor stability and short half-life in vivo. Rao et $\mathrm{al}^{114}$ developed chitosan-decorated SeNPs as its carriers to improve the in vivo half-life, hence the curative effect on type 2 DM. BAY 55-983-loaded SeNPs displayed good stability and a high protein-loading rate, and the release of BAY 55-9837 from SeNPs lasted for over 70 hours with the accumulative release percentage of $78.9 \%$. Moreover, the conjugation of BAY 55-9837 with SeNPs significantly reduced its renal clearance to a rate of $1.56 \mathrm{~mL} / \mathrm{h}$ and extended its half-life to 20.81 hours. The authors argued that amplification in the apparent molecular size through nanocomplexation could reduce the renal elimination, which may be beneficial for treating type $2 \mathrm{DM}$ plus the antidiabetic effect of SeNPs themselves. As a follow-up study, the research team constructed novel peptide-conjugated SeNPs comprising a 


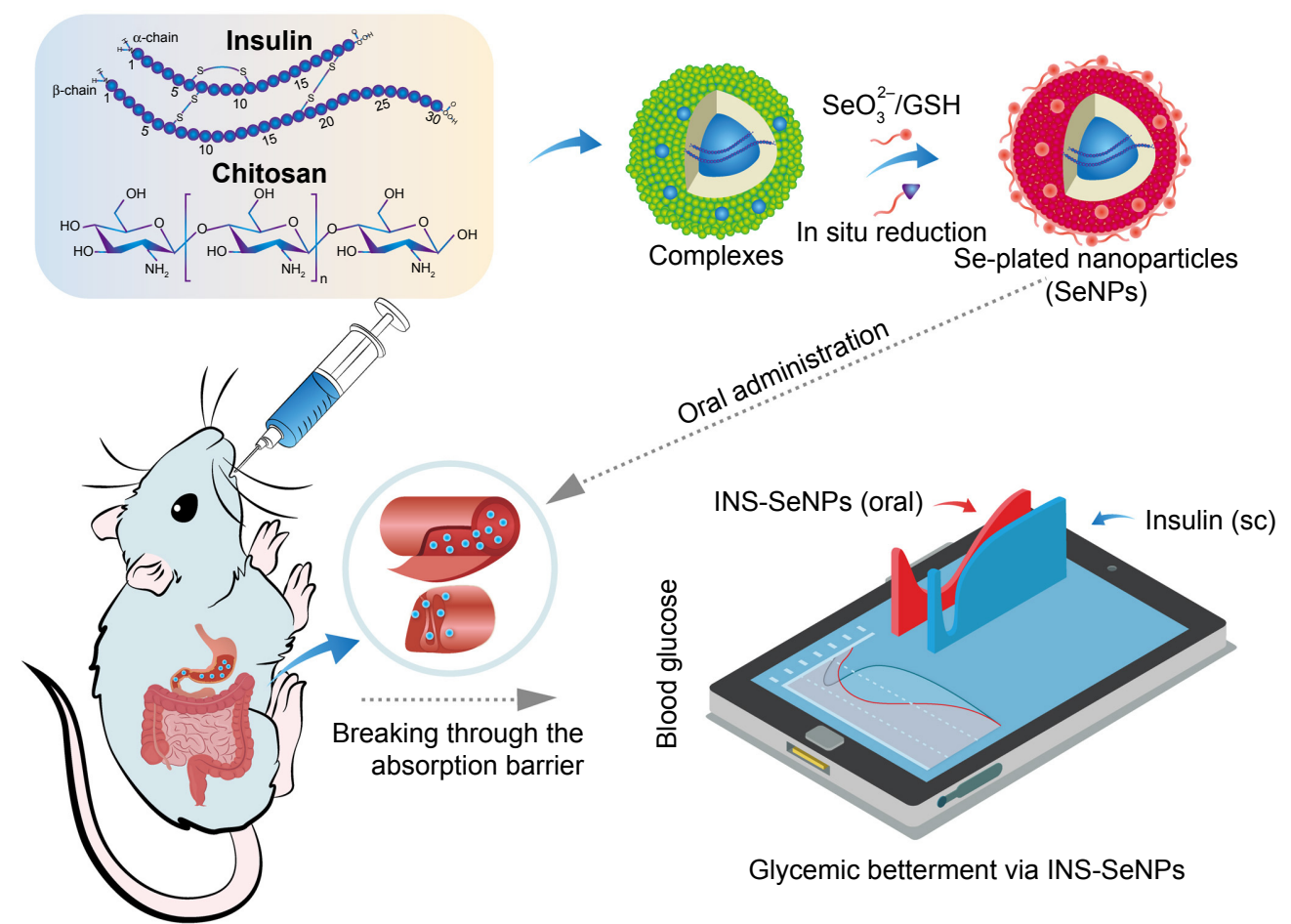

Figure 5 Schematic illustration of INS-SeNPs' preparation, absorption feature, and hypoglycemic effect delivered via the oral route.

Note: Reprinted from Nanomedicine, 13(6), Deng W, Xie Q, Wang H, Ma Z, Wu B, Zhang X, Selenium nanoparticles as versatile carriers for oral delivery of insulin: Insight into the synergic antidiabetic effect and mechanism, 1965-1974, Copyright 2017, with permission from Elsevier. ${ }^{113}$

Abbreviations: INS-SeNPs, insulin-loaded SeNPs; sc, subcutaneous; SeNPs, selenium nanoparticles.

32-amino acid peptide specific to VPAC2 to deliver pituitary adenylate cyclase-activating peptide (PACAP), a novel neuroendocrine peptide. ${ }^{115}$ PACAP-loaded SeNPs with a peptide ligand significantly promoted INS-1 cell proliferation, glucose uptake, insulin secretion, and insulin receptor expression and also markedly reduced intracellular ROS levels in $\mathrm{H}_{2} \mathrm{O}_{2}$-injured INS-1 cells. As such, PACAP-loaded SeNPs resulted in enhanced insulin secretion and sustained hypoglycemic effect at an injection dose of $20 \mathrm{nmol} / \mathrm{L}$ in the type $2 \mathrm{DM} \mathrm{db} / \mathrm{db}$ mice. Repeated administration for 12 weeks significantly improved the glucose and lipid profiles, insulin sensitivity, and the histomorphology of pancreatic and adipose tissues. These results indicate that SeNPs as carriers of bioactive peptides can sustain their release and append them a synergic effect on treatment.

\section{Nucleic acid drugs}

Nucleic acid drugs refer to functional nucleotides with therapeutic purposes, including aptamers, antisense oligonucleotide, siRNA, and other RNA inhibitors, mimics, and modulators. ${ }^{116}$ Nucleic acid drugs can cure various genetic diseases at the gene level, thus known as gene therapy. Abundant charge and ionic groups enable nucleic acids to attach to SeNPs, which establishes the basis of SeNPloading nucleic acids. Drug loading with SeNPs mainly takes advantage of the synergic effect of SeNPs with the cargo. The potentiation of SeNPs to therapeutic agents is equally exemplified by siRNA designed for tumor targeting therapy. To obtain a high therapeutic efficacy, Zheng et al ${ }^{117}$ employed layered double hydroxides (LDHs) to simultaneously deliver selenium and pooled siRNA. LDH-supported SeNPs (Se@LDH) were compacted with siRNAs (anti-P-gp and anti- $\beta$-tubulin III) via electrostatic interactions. A series of siRNA transfection experiments confirmed that a higher gene silencing efficiency was induced by Se@LDH than LDH. Se@LDH exhibited a microtubule-stabilizing effect that inhibited cell proliferation by blocking cell cycle at the G2/M phase, disrupting normal mitotic spindle formation, and inducing cell apoptosis. Moreover, pooled siRNAloaded Se@LDH induced cell apoptosis, transformed cell morphology, and raised cellular ROS levels by regulating the expression of Bcl-2/Bax, activating caspase-3, PI3K/AKT/ mTOR, and MAPK/ERK pathways. In Li et al's research, ${ }^{118}$ polyethylenimine (PEI)-modified SeNPs were proposed for formulating siRNA to silence inducible HSP70, a heat shock protein, to enhance the apoptosis of HepG2 cells. SeNPs loading siRNA against HSP70 (SeNPs@siRNA) showed satisfactory size distribution, high stability, and selectivity between cancer and normal cells. The cytotoxicity of SeNPs@siRNA was lower for normal cells than 
for tumor cells. Of note, the gene-silencing efficiency of SeNPs@siRNA was significantly higher than Lipofectamine 2000@siRNA and resulted in a distinct reduction in HSP70 mRNA and protein expression in cancer cells. It was shown that intracellular ROS overproduction and activation of p53 and AKT signaling pathways were responsible for enhanced cytotoxic effects. Altogether, these studies provide insight into the use of SeNPs as effective carriers of nucleic acid drugs, which may be hopeful in gene therapy.

\section{Co-delivery of cytotoxic agents and siRNA} Co-delivery or dual delivery has emerged as a promising mode in oncotherapy. The versatility of SeNPs allows different therapeutic cargos to be loaded and delivered to the action sites. Co-delivery of cytotoxic agents and therapeutic siRNA through multifunctional nanoparticles has become as an appealing treatment modality. SeNPs have been explored for co-delivery of Dox and siRNA to enhance the cancer therapy. ${ }^{119}$ In one study, RGDfC peptide as a tumor-targeting moiety was modified onto SeNPs by an adsorption effect, following which Dox and siRNA were loaded onto SeNPs by electrostatic interaction. RGDfC-SeNPs@Dox/siRNA could readily enter into HepG2 cells by clathrin-associated endocytosis and showed high gene transfection efficiency. The in vivo studies indicated that such nanoparticles accumulated more in the tumor site and resulted in a more significant anticancer activity than any individual component. Multidrug resistance constitutes a great barrier against effective cancer killing. To overcome and reverse drug resistance in tumor cells, Zheng et al ${ }^{120}$ devised PEI-modified SeNPs for co-delivery of cisplatin and mdr1 siRNA. SeNPs exhibited a high siRNA loading and gene-silencing efficacy. They caused downregulation of P-glycoprotein and multidrug resistance-associated protein expression. The co-delivery system elevated the cytotoxicity of cisplatin in A549 cells resistant to cisplatin by apoptosis induction through the AKT and ERK signaling pathways. Animal studies also demonstrated that the co-delivery system effectively enhanced the anticancer effect on tumor-bearing nude mice. These cases indicate that SeNPs can integrate chemotherapy and gene therapy into one module to corporately defeat intractable malignant diseases.

\section{Conclusion}

Selenium as a pleiotropic nutrient has sparked tremendous interests for the investigation of various biomedical purposes. Selenium takes possession of a number of physiological functions and plays a pivotal role in life activities. However, the physiological effects are closely associated with the concentration and modality of selenium. This requires a precise dose settlement for selenium so as to properly exert its pharmacological actions. With the ease of chemical modification, selenium can be processed into special medications with good performance. SeNPs as a matter of nano-Se represent the advanced form of selenium therapeuticals that exhibit lower toxicity to normal tissues and higher efficacy to diseased tissues owing to superb pharmacokinetics and selectivity. SeNPs have been extensively explored for their potential in disease intervention and drug delivery, showing encouraging outcomes in antitumor, antidiabetes as well as other Se-associated diseases. Se-based nanomedicines are shedding light on the utilization of a multifunctional agent to achieve a precise therapy.

\section{Acknowledgments}

This work was jointly supported by the National Natural Science Foundation of China (81673604), Fundamental Research Funds for the Central Universities (21617473 and 21615473), Scientific Research Foundation of the First Affiliated Hospital of Jinan University, China (2015210 and 2017305), Medical Science and Technology Foundation of Guangdong Province (A2018313 and A2016040), Traditional Chinese Medicine Administration Foundation of Guangdong Province (20161072), and Natural Science Foundation of Guangdong Province (2016ZC0042 and 2016ZC0048).

\section{Disclosure}

The authors report no conflicts of interest in this work.

\section{References}

1. Davy T, Castellano S. The genomics of selenium: Its past, present and future. Biochim Biophys Acta Gen Subj. Epub 2018 May 30.

2. Duntas LH, Benvenga S. Selenium: an element for life. Endocrine. 2015; 48(3):756-775.

3. Romero-Pérez A, García-García E, Zavaleta-Mancera A, et al. Designing and evaluation of sodium selenite nanoparticles in vitro to improve selenium absorption in ruminants. Vet Res Commun. 2010;34(1):71-79.

4. Kieliszek M, Błażejak S. Selenium: Significance, and outlook for supplementation. Nutrition. 2013;29(5):713-718.

5. Kristal AR, Darke AK, Morris JS, et al. Baseline selenium status and effects of selenium and vitamin e supplementation on prostate cancer risk. J Natl Cancer Inst. 2014;106(3):djt456.

6. Gharipour M, Sadeghi M, Behmanesh M, Salehi M, Nezafati P, Gharpour A. Selenium homeostasis and clustering of cardiovascular risk factors: a systematic review. Acta Biomed. 2017;88(3):263-270.

7. Tajaddini MH, Keikha M, Razzazzadeh A, Kelishadi R. A systematic review on the association of serum selenium and metabolic syndrome. J Res Med Sci. 2015;20(8):782-789.

8. Vinceti M, Filippini T, Cilloni S, Crespi CM. The Epidemiology of selenium and human cancer. Adv Cancer Res. 2017;136:1-48. 
9. Wojciechowska-Durczynska K, Lewinski A. Search for relevant indications for selenium supplementation in thyroid diseases. Neuro Endocrinol Lett. 2017;38(4):237-241.

10. Fang X, Li C, Zheng L, Yang F, Chen T. Dual-targeted selenium nanoparticles for synergistic photothermal therapy and chemotherapy of tumors. Chem Asian J. 2018;13(8):996-1004.

11. Zhai S, Hu X, Hu Y, Wu B, Xing D. Visible light-induced crosslinking and physiological stabilization of diselenide-rich nanoparticles for redox-responsive drug release and combination chemotherapy. Biomaterials. 2017;121:41-54.

12. Younus H. Therapeutic potentials of superoxide dismutase. Int J Health Sci. 2018;12(3):88-93.

13. Labunskyy VM, Hatfield DL, Gladyshev VN. Selenoproteins: molecular pathways and physiological roles. Physiol Rev. 2014;94(3):739-777.

14. Bellinger FP, Raman AV, Reeves MA, Berry MJ. Regulation and function of selenoproteins in human disease. Biochem J. 2009;422(1):11-22.

15. Maroney MJ, Hondal RJ. Selenium versus sulfur: Reversibility of chemical reactions and resistance to permanent oxidation in proteins and nucleic acids. Free Radic Biol Med. 2018;127:228-237.

16. Álvarez-Pérez M, Ali W, Marć MA, Handzlik J, Domínguez-Álvarez E. Selenides and diselenides: a review of their anticancer and chemopreventive activity. Molecules. 2018;23(3):628.

17. Collery P. Strategies for the development of selenium-based anticancer drugs. J Trace Elem Med Biol. 2018;50:498-507.

18. Meinhold H, Campos-Barros A, Walzog B, Köhler R, Müller F, Behne D. Effects of selenium and iodine deficiency on type I, type II and type III iodothyronine deiodinases and circulating thyroid hormones in the rat. Exp Clin Endocrinol. 1993;101(2):87-93.

19. Arthur JR, Beckett GJ, Mitchell JH. The interactions between selenium and iodine deficiencies in man and animals. Nutr Res Rev. 1999; 12(1):55-73.

20. Zhou J, Huang K, Lei XG. Selenium and diabetes-evidence from animal studies. Free Radic Biol Med. 2013;65:1548-1556.

21. Broome CS, Mcardle F, Kyle JA, et al. An increase in selenium intake improves immune function and poliovirus handling in adults with marginal selenium status. Am J Clin Nutr. 2004;80(1):154-162.

22. Beck MA, Handy J, Levander OA. Host nutritional status: the neglected virulence factor. Trends Microbiol. 2004;12(9):417-423.

23. Aaseth J, Alexander J, Bjørklund G, et al. Treatment strategies in Alzheimer's disease: a review with focus on selenium supplementation. Biometals. 2016;29(5):827-839.

24. Moslemi MK, Tavanbakhsh S. Selenium-vitamin E supplementation in infertile men: effects on semen parameters and pregnancy rate. Int J Gen Med. 2011;4:99-104.

25. Conner TS, Richardson AC, Miller JC. Optimal serum selenium concentrations are associated with lower depressive symptoms and negative mood among young adults. J Nutr. 2015;145(1):59-65.

26. Thompson JM, Mirza MA, Park MK, Qureshi AA, Cho E. The role of micronutrients in alopecia areata: a review. Am J Clin Dermatol. 2017; 18(5):663-679.

27. Loscalzo J, Disease K. Keshan disease, selenium deficiency, and the selenoproteome. N Engl J Med. 2014;370(18):1756-1760.

28. Lorenzo Alonso MJ, Bermejo Barrera A, Cocho de Juan JA, Fraga Bermúdez JM, Bermejo Barrera P. Selenium levels in related biological samples: human placenta, maternal and umbilical cord blood, hair and nails. J Trace Elem Med Biol. 2005;19(1):49-54.

29. Fairweather-Tait SJ, Collings R, Hurst R. Selenium bioavailability: current knowledge and future research requirements. Am J Clin Nutr. 2010;91(5):1484S-1491S.

30. Hosnedlova B, Kepinska M, Skalickova S, et al. Nano-selenium and its nanomedicine applications: a critical review. Int J Nanomedicine. 2018;13:2107-2128.

31. Ramoutar RR, Brumaghim JL. Antioxidant and anticancer properties and mechanisms of inorganic selenium, oxo-sulfur, and oxo-selenium compounds. Cell Biochem Biophys. 2010;58(1):1-23.

32. Sieja K, Talerczyk M. Selenium as an element in the treatment of ovarian cancer in women receiving chemotherapy. Gynecol Oncol. 2004;93(2): $320-327$.
33. Lee KH, Jeong D. Bimodal actions of selenium essential for antioxidant and toxic pro-oxidant activities: the selenium paradox (Review). Mol Med Rep. 2012;5(2):299-304.

34. Wallenberg M, Misra S, Björnstedt M. Selenium cytotoxicity in cancer. Basic Clin Pharmacol Toxicol. 2014;114(5):377-386.

35. Bhise K, Sau S, Alsaab H, Kashaw SK, Tekade RK, Iyer AK. Nanomedicine for cancer diagnosis and therapy: advancement, success and structure-activity relationship. Ther Deliv. 2017;8(11):1003-1018.

36. Chen T, Wong YS, Zheng W, Bai Y, Huang L. Selenium nanoparticles fabricated in Undaria pinnatifida polysaccharide solutions induce mitochondria-mediated apoptosis in A375 human melanoma cells. Colloids Surf B Biointerfaces. 2008;67(1):26-31.

37. Menon S, Ks SD, R S, S R, S VK. Selenium nanoparticles: A potent chemotherapeutic agent and an elucidation of its mechanism. Colloids Surf B Biointerfaces. 2018;170:280-292.

38. Liu T, Zeng L, Jiang W, Fu Y, Zheng W, Chen T. Rational design of cancer-targeted selenium nanoparticles to antagonize multidrug resistance in cancer cells. Nanomedicine. 2015;11(4):947-958.

39. Sonkusre P, Cameotra SS. Biogenic selenium nanoparticles induce ROS-mediated necroptosis in PC-3 cancer cells through TNF activation. J Nanobiotechnology. 2017;15(1):43.

40. Shahverdi AR, Shahverdi F, Faghfuri E, et al. Characterization of Folic Acid Surface-Coated Selenium Nanoparticles and Corresponding In Vitro and In Vivo Effects Against Breast Cancer. Arch Med Res. 2018;49(1):10-17.

41. Cui D, Yan C, Miao J, et al. Synthesis, characterization and antitumor properties of selenium nanoparticles coupling with ferulic acid. Mater Sci Eng C Mater Biol Appl. 2018;90:104-112.

42. Zhao S, Yu Q, Pan J, et al. Redox-responsive mesoporous selenium delivery of doxorubicin targets MCF-7 cells and synergistically enhances its anti-tumor activity. Acta Biomater. 2017;54:294-306.

43. Feng Y, Su J, Zhao Z, et al. Differential effects of amino acid surface decoration on the anticancer efficacy of selenium nanoparticles. Dalton Trans. 2014;43(4):1854-1861.

44. Alam U, Asghar O, Azmi S, Malik RA. General aspects of diabetes mellitus. Handb Clin Neurol. 2014;126:211-222.

45. Akbaraly TN, Arnaud J, Rayman MP, et al. Plasma selenium and risk of dysglycemia in an elderly French population: results from the prospective Epidemiology of Vascular Ageing Study. Nutr Metab. 2010; $7(1): 21$.

46. Park K, Rimm EB, Siscovick DS, et al. Toenail selenium and incidence of type 2 diabetes in U.S. men and women. Diabetes Care. 2012;35(7): 1544-1551.

47. Galan-Chilet I, Grau-Perez M, de Marco G, et al. A gene-environment interaction analysis of plasma selenium with prevalent and incident diabetes: The Hortega study. Redox Biol. 2017;12:798-805.

48. Yuan Y, Xiao Y, Yu Y, et al. Associations of multiple plasma metals with incident type 2 diabetes in Chinese adults: The Dongfeng-Tongji Cohort. Environ Pollut. 2018;237:917-925.

49. Muriach M, Flores-Bellver M, Romero FJ, Barcia JM. Diabetes and the brain: oxidative stress, inflammation, and autophagy. Oxid Med Cell Longev. 2014;2014(2).

50. Gao Z, Li J, Song X, et al. Antioxidative, anti-inflammation and lung-protective effects of mycelia selenium polysaccharides from Oudemansiella radicata. Int J Biol Macromol. 2017;104(Pt A): 1158-1164.

51. Rayman MP. Selenium and human health. Lancet. 2012;379(9822): 1256-1268.

52. Cancarini A, Fostinelli J, Napoli L, Gilberti ME, Apostoli P, Semeraro F. Trace elements and diabetes: Assessment of levels in tears and serum. Exp Eye Res. 2017;154:47-52.

53. Zhang H, Yan C, Yang Z, et al. Alterations of serum trace elements in patients with type 2 diabetes. J Trace Elem Med Biol. 2017;40:91-96.

54. Brownlee M. Biochemistry and molecular cell biology of diabetic complications. Nature. 2001;414(6865):813-820.

55. Al-Quraishy S, Dkhil MA, Abdel Moneim AE. Anti-hyperglycemic activity of selenium nanoparticles in streptozotocin-induced diabetic rats. Int J Nanomedicine. 2015;10:6741-6756. 
56. Liu Y, Zeng S, Liu Y, et al. Synthesis and antidiabetic activity of selenium nanoparticles in the presence of polysaccharides from Catathelasma ventricosum. Int J Biol Macromol. 2018;114:632-639.

57. Zeng S, Ke Y, Liu Y, et al. Synthesis and antidiabetic properties of chitosan-stabilized selenium nanoparticles. Colloids Surf B Biointerfaces. 2018;170:115-121.

58. Ahmed HH, Abd El-Maksoud MD, Abdel Moneim AE, Aglan HA. Preclinical study for the antidiabetic potential of selenium nanoparticles. Biol Trace Elem Res. 2017;177(2):267-280.

59. Chaudhary S, Umar A, Mehta SK. Surface functionalized selenium nanoparticles for biomedical applications. J Biomed Nanotechnol. 2014; 10(10):3004-3042.

60. Zhu C, Zhang S, Song C, et al. Selenium nanoparticles decorated with Ulva lactuca polysaccharide potentially attenuate colitis by inhibiting

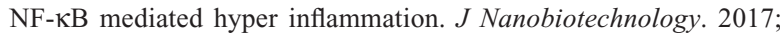
15(1):20.

61. Alkhudhayri AA, Dkhil MA, Al-Quraishy S. Nanoselenium prevents eimeriosis-induced inflammation and regulates mucin gene expression in mice jejunum. Int J Nanomedicine. 2018;13:1993-2003.

62. Zhai X, Zhang C, Zhao G, Stoll S, Ren F, Leng X. Antioxidant capacities of the selenium nanoparticles stabilized by chitosan. J Nanobiotechnology. 2017;15(1):4.

63. Malhotra S, Welling MN, Mantri SB, Desai K. In vitro and in vivo antioxidant, cytotoxic, and anti-chronic inflammatory arthritic effect of selenium nanoparticles. J Biomed Mater Res B Appl Biomater. 2016; 104(5):993-1003.

64. Srivastava N, Mukhopadhyay M. Green synthesis and structural characterization of selenium nanoparticles and assessment of their antimicrobial property. Bioprocess Biosyst Eng. 2015;38(9):1723-1730.

65. Lara HH, Guisbiers G, Mendoza J, et al. Synergistic antifungal effect of chitosan-stabilized selenium nanoparticles synthesized by pulsed laser ablation in liquids against Candida albicans biofilms. Int $J$ Nanomedicine. 2018;13:2697-2708.

66. Li Y, Lin Z, Guo M, et al. Inhibitory activity of selenium nanoparticles functionalized with oseltamivir on H1N1 influenza virus. Int J Nanomedicine. 2017;12:5733-5743.

67. Li Y, Lin Z, Guo M, et al. Inhibition of H1N1 influenza virus-induced apoptosis by functionalized selenium nanoparticles with amantadine through ROS-mediated AKT signaling pathways. Int J Nanomedicine. 2018;13:2005-2016.

68. Stuss M, Michalska-Kasiczak M, Sewerynek E. The role of selenium in thyroid gland pathophysiology. Endokrynol Pol. 2017;68(4):440-465.

69. Atteia HH, Arafa MH, Prabahar K. Selenium nanoparticles prevents lead acetate-induced hypothyroidism and oxidative damage of thyroid tissues in male rats through modulation of selenoenzymes and suppression of miR-224. Biomed Pharmacother. 2018;99:486-491.

70. Nazıroğlu M, Muhamad S, Pecze L. Nanoparticles as potential clinical therapeutic agents in Alzheimer's disease: focus on selenium nanoparticles. Expert Rev Clin Pharmacol. 2017;10(7):773-782.

71. Yin T, Yang L, Liu Y, Zhou X, Sun J, Liu J. Sialic acid (SA)-modified selenium nanoparticles coated with a high blood-brain barrier permeability peptide-B6 peptide for potential use in Alzheimer's disease. Acta Biomater. 2015;25:172-183.

72. Muecke R, Micke O, Schomburg L, et al. Selenium in Radiation Oncology-15 Years of Experiences in Germany. Nutrients. 2018; 10(4):E483.

73. Chan L, He L, Zhou B, et al. Cancer-Targeted Selenium Nanoparticles Sensitize Cancer Cells to Continuous $\gamma$ Radiation to Achieve Synergetic Chemo-Radiotherapy. Chem Asian J. 2017;12(23):3053-3060.

74. Yang Y, Xie Q, Zhao Z, et al. Functionalized Selenium Nanosystem as Radiation Sensitizer of ${ }^{125}$ I Seeds for Precise Cancer Therapy. ACS Appl Mater Interfaces. 2017;9(31):25857-25869.

75. Abd-Rabou AA, Shalby AB, Ahmed HH. Selenium nanoparticles induce the chemo-sensitivity of fluorouracil nanoparticles in breast and colon cancer cells. Biol Trace Elem Res. 2018;20(8).

76. Faghfuri E, Yazdi MH, Mahdavi M, et al. Dose-response relationship study of selenium nanoparticles as an immunostimulatory agent in cancer-bearing mice. Arch Med Res. 2015;46(1):31-37.
77. Gao F, Yuan Q, Gao L, et al. Cytotoxicity and therapeutic effect of irinotecan combined with selenium nanoparticles. Biomaterials. 2014; 35(31):8854-8866.

78. Ramamurthy C, Sampath KS, Arunkumar P, et al. Green synthesis and characterization of selenium nanoparticles and its augmented cytotoxicity with doxorubicin on cancer cells. Bioprocess Biosyst Eng. 2013;36(8):1131-1139.

79. Bhattacharjee A, Basu A, Biswas J, Sen T, Bhattacharya S. Chemoprotective and chemosensitizing properties of selenium nanoparticle (Nano-Se) during adjuvant therapy with cyclophosphamide in tumorbearing mice. Mol Cell Biochem. 2017;424(1-2):13-33.

80. Li Y, Li X, Wong YS, et al. The reversal of cisplatin-induced nephrotoxicity by selenium nanoparticles functionalized with 11-mercapto1-undecanol by inhibition of ROS-mediated apoptosis. Biomaterials. 2011;32(34):9068-9076.

81. Rezvanfar MA, Rezvanfar MA, Shahverdi AR, et al. Protection of cisplatin-induced spermatotoxicity, DNA damage and chromatin abnormality by selenium nano-particles. Toxicol Appl Pharmacol. 2013; 266(3):356-365.

82. Sadek KM, Lebda MA, Abouzed TK, Nasr SM, Shoukry M. Neuroand nephrotoxicity of subchronic cadmium chloride exposure and the potential chemoprotective effects of selenium nanoparticles. Metab Brain Dis. 2017;32(5):1659-1673.

83. Ungvári É, Monori I, Megyeri A, et al. Protective effects of meat from lambs on selenium nanoparticle supplemented diet in a mouse model of polycyclic aromatic hydrocarbon-induced immunotoxicity. Food Chem Toxicol. 2014;64:298-306.

84. Kojouri GA, Jahanabadi S, Shakibaie M, Ahadi AM, Shahverdi AR. Effect of selenium supplementation with sodium selenite and selenium nanoparticles on iron homeostasis and transferrin gene expression in sheep: a preliminary study. Res Vet Sci. 2012;93(1):275-278.

85. Prasad KS, Selvaraj K. Biogenic synthesis of selenium nanoparticles and their effect on As(III)-induced toxicity on human lymphocytes. Biol Trace Elem Res. 2014;157(3):275-283.

86. Hassanin KM, Abd El-Kawi SH, Hashem KS. The prospective protective effect of selenium nanoparticles against chromium-induced oxidative and cellular damage in rat thyroid. Int J Nanomedicine. 2013; 8:1713-1720.

87. Trabelsi H, Azzouz I, Ferchichi S, Tebourbi O, Sakly M, Abdelmelek H. Nanotoxicological evaluation of oxidative responses in rat nephrocytes induced by cadmium. Int J Nanomedicine. 2013;8:3447-3453.

88. Zhao Y, Sun Q, Zhang X, Baeyens J, Su H. Self-assembled selenium nanoparticles and their application in the rapid diagnostic detection of small cell lung cancer biomarkers. Soft Matter. 2018;14(4): $481-489$.

89. Khalid A, Tran PA, Norello R, Simpson DA, O'Connor AJ, TomljenovicHanic S. Intrinsic fluorescence of selenium nanoparticles for cellular imaging applications. Nanoscale. 2016;8(6):3376-3385.

90. Wang T, Yang L, Zhang B, Liu J. Extracellular biosynthesis and transformation of selenium nanoparticles and application in $\mathrm{H}_{2} \mathrm{O}_{2}$ biosensor. Colloids Surf B Biointerfaces. 2010;80(1):94-102.

91. Mistry HD, Broughton Pipkin F, Redman CW, Poston L. Selenium in reproductive health. Am J Obstet Gynecol. 2012;206(1):21-30.

92. Liu L, He Y, Xiao Z, et al. Effects of selenium nanoparticles on reproductive performance of male Sprague-Dawley rats at supranutritional and nonlethal levels. Biol Trace Elem Res. 2017;180(1):81-89.

93. Wu X, Yao J, Yang Z, et al. Improved fetal hair follicle development by maternal supplement of selenium at nano size (Nano-Se). Livest Sci. 2011;142(1-3):270-275.

94. Han J, Guo X, Lei Y, Dennis BS, Wu S, Wu C. Synthesis and characterization of selenium-chondroitin sulfate nanoparticles. Carbohydr Polym. 2012;90(1):122-126.

95. Beheshti N, Soflaei S, Shakibaie M, et al. Efficacy of biogenic selenium nanoparticles against Leishmania major: in vitro and in vivo studies. J Trace Elem Med Biol. 2013;27(3):203-207.

96. Mahmoudvand H, Fasihi Harandi M, Shakibaie M, et al. Scolicidal effects of biogenic selenium nanoparticles against protoscolices of hydatid cysts. Int J Surg. 2014;12(5):399-403. 
97. Amin KA, Hashem KS, Alshehri FS, Awad ST, Hassan MS. Antioxidant and hepatoprotective efficiency of selenium nanoparticles against acetaminophen-induced hepatic damage. Biol Trace Elem Res. 2017; 175(1):136-145.

98. Kumar GS, Kulkarni A, Khurana A, Kaur J, Tikoo K. Selenium nanoparticles involve HSP-70 and SIRT1 in preventing the progression of type 1 diabetic nephropathy. Chem Biol Interact. 2014;223: 125-133.

99. Maiyo F, Singh M. Selenium nanoparticles: potential in cancer gene and drug delivery. Nanomedicine. 2017;12(9):1075-1089.

100. Dwivedi C, Shah CP, Singh K, Kumar M, Bajaj PN. An organic acidinduced synthesis and characterization of selenium nanoparticles. J Nanotechnol. 2011;2011(2):1-6.

101. Yin J, Hou Y, Yin Y, Song X. Selenium-coated nanostructured lipid carriers used for oral delivery of berberine to accomplish a synergic hypoglycemic effect. Int J Nanomedicine. 2017;12:8671-8680.

102. Wen R, Banik B, Pathak RK, Kumar A, Kolishetti N, Dhar S. Nanotechnology inspired tools for mitochondrial dysfunction related diseases. Adv Drug Deliv Rev. 2016;99(Pt A):52-69.

103. Hou J, Yu X, Shen Y, Shi Y, Su C, Zhao L. Triphenyl phosphinefunctionalized chitosan nanoparticles enhanced antitumor efficiency through targeted delivery of doxorubicin to mitochondria. Nanoscale Res Lett. 2017;12(1):158.

104. Jameson VJ, Cochemé HM, Logan A, Hanton LR, Smith RA, Murphy MP. Synthesis of triphenylphosphonium vitamin E derivatives as mitochondria-targeted antioxidants. Tetrahedron. 2015;71(44): 8444-8453.

105. Kumari M, Ray L, Purohit MP, et al. Curcumin loading potentiates the chemotherapeutic efficacy of selenium nanoparticles in HCT116 cells and Ehrlich's ascites carcinoma bearing mice. Eur J Pharm Biopharm. 2017;117:346-362.

106. Bidkar AP, Sanpui P, Ghosh SS. Efficient induction of apoptosis in cancer cells by paclitaxel-loaded selenium nanoparticles. Nanomedicine. 2017;12(21):2641-2651.

107. Pi J, Jiang J, Cai H, et al. GE11 peptide conjugated selenium nanoparticles for EGFR targeted oridonin delivery to achieve enhanced anticancer efficacy by inhibiting EGFR-mediated PI3K/ AKT and Ras/Raf/MEK/ERK pathways. Drug Deliv. 2017;24(1): 1549-1564.

108. Jalalian SH, Ramezani M, Abnous K, Taghdisi SM. Targeted codelivery of epirubicin and NAS-24 aptamer to cancer cells using selenium nanoparticles for enhancing tumor response in vitro and in vivo. Cancer Lett. 2018;416:87-93.
109. Xie Q, Deng W, Yuan X, et al. Selenium-functionalized liposomes for systemic delivery of doxorubicin with enhanced pharmacokinetics and anticancer effect. Eur J Pharm Biopharm. 2018;122:87-95.

110. Zhang J, Teng Z, Yuan Y, et al. Development, physicochemical characterization and cytotoxicity of selenium nanoparticles stabilized by betalactoglobulin. Int J Biol Macromol. 2018;107(Pt B):1406-1413.

111. Borovikova LN, Titova AV, Matveeva NA, Pisarev OA. Stabilizing selenium nanoparticles with chymotrypsin: The effect of $\mathrm{pH}$ and nanoparticle-enzyme concentration ratios on the stability of nanocomplexes. Russian J Phys Chem A. 2013;87(6):998-1001.

112. Xu D, Yang L, Wang Y, Wang G, Rensing C, Zheng S. Proteins enriched in charged amino acids control the formation and stabilization of selenium nanoparticles in Comamonas testosteroni S44. Sci Rep. 2018;8(1):4766.

113. Deng W, Xie Q, Wang H, Ma Z, Wu B, Zhang X. Selenium nanoparticles as versatile carriers for oral delivery of insulin: Insight into the synergic antidiabetic effect and mechanism. Nanomedicine. 2017;13(6): 1965-1974.

114. Rao L, Ma Y, Zhuang M, Luo T, Wang Y, Hong A. Chitosan-decorated selenium nanoparticles as protein carriers to improve the in vivo half-life of the peptide therapeutic BAY 55-9837 for type 2 diabetes mellitus. Int J Nanomedicine. 2014;9:4819-4828.

115. Zhao SJ, Wang DH, Li YW, et al. A novel selective VPAC2 agonist peptide-conjugated chitosan modified selenium nanoparticles with enhanced anti-type 2 diabetes synergy effects. Int J Nanomedicine. 2017;12:2143-2160.

116. Chen $C$, Yang Z, Tang X. Chemical modifications of nucleic acid drugs and their delivery systems for gene-based therapy. Med Res Rev. 2018;38(3):829-869.

117. Zheng W, Yin T, Chen Q, et al. Co-delivery of Se nanoparticles and pooled SiRNAs for overcoming drug resistance mediated by P-glycoprotein and class III $\beta$-tubulin in drug-resistant breast cancers. Acta Biomater. 2016;31:197-210.

118. Li Y, Lin Z, Zhao M, et al. Multifunctional selenium nanoparticles as carriers of HSP70 siRNA to induce apoptosis of HepG2 cells. Int J Nanomedicine. 2016;11:3065-3076.

119. Xia Y, Xu T, Wang C, et al. Novel functionalized nanoparticles for tumor-targeting co-delivery of doxorubicin and siRNA to enhance cancer therapy. Int J Nanomedicine. 2018;13:143-159.

120. Zheng W, Cao C, Liu Y, et al. Multifunctional polyamidoaminemodified selenium nanoparticles dual-delivering siRNA and cisplatin to A549/DDP cells for reversal multidrug resistance. Acta Biomater. 2015;11:368-380
International Journal of Nanomedicine

\section{Publish your work in this journal}

The International Journal of Nanomedicine is an international, peerreviewed journal focusing on the application of nanotechnology in diagnostics, therapeutics, and drug delivery systems throughout the biomedical field. This journal is indexed on PubMed Central, MedLine, CAS, SciSearch ${ }^{\circledR}$, Current Contents ${ }^{\circledR} /$ Clinical Medicine,

\section{Dovepress}

Journal Citation Reports/Science Edition, EMBase, Scopus and the Elsevier Bibliographic databases. The manuscript management system is completely online and includes a very quick and fair peer-review system, which is all easy to use. Visit http://www.dovepress.com/ testimonials.php to read real quotes from published authors. 